\title{
A free boundary problem arising in the ecological models with $N$-species
}

Qi-Jian $\operatorname{Tan}^{*}$

\section{"Correspondence:} tanqjxxx@163.com

Department of Mathematics, Chengdu Normal University,

Chengdu, 611130, P.R. China

\section{Springer}

\begin{abstract}
This paper is concerned with the one-dimensional free boundary problem for quasilinear reaction-diffusion systems arising in the ecological models with $\mathrm{N}$-species, where some of the species are made up of two separated groups and the mankind?s influence is taken into account. In the problem under consideration, there are $n$ free boundaries, the coefficients of the equations are allowed to be discontinuous on the free boundaries and the reaction functions are mixed quasimonotone. The aim is to show the local existence of the solutions for the free boundary problem by the fixed point method, and the global existence and uniqueness of the solutions for the corresponding diffraction problem by the approximation and estimate methods. MSC: 35R35; 35R05; 35K57; 35K20
\end{abstract}

Keywords: free boundary; diffraction problem; reaction-diffusion system; existence; fixed point theorem; approximation method

\section{Introduction}

For some animal species, different groups may live in different habitats separated by free boundaries (see [1]). For the case of one-species and one-dimensional, Kim and Lin [1] proposed a model to describe the evolution of the free boundary. In this paper, we consider the one-dimensional ecological models with $N$-species where the mankind?s influence is taken into account. Assume that there are $N$ species sharing the same habitat, the onedimensional domain $(0, d)$. For some $1 \leq n \leq N$, assume further that each of the $n$ species is divided into two groups, whereas each of the rest of $N-n$ species has a single group.

To describe the free boundary problem, we introduce some notations:

$Q_{T}:=(0, d) \times(0, T]$ is the open rectangle (and upper horizontal line).

$\bar{Q}_{T}$ is the entire closed rectangle.

$S_{T}$ is made up of the closed left and right boundaries of $Q_{T}$.

$\Gamma_{T}^{l}:=\left\{(x, t): x=\varphi^{l}(t), 0 \leq t \leq T\right\}$ is the free boundary for species $l, l \in\{1, \ldots, n\}$.

$Q_{T}^{l-}:=\left\{(x, t): 0<x<\varphi^{l}(t), 0<t \leq T\right\}, Q_{T}^{l_{+}}:=\left\{(x, t): \varphi^{l}(t)<x<d, 0<t \leq T\right\}$ are the

subdomains of $Q_{T}$ separated by $\Gamma_{T}^{l}, l \in\{1, \ldots, n\}$.

$Q_{k, T}:=\left\{(x, t): \varphi^{k-1}(t)<x<\varphi^{k}(t), 0<t \leq T\right\}$ is the region between consecutive

boundaries, $k \in\{1, \ldots, n+1\}$, where $\varphi^{0}(t) \equiv 0, \varphi^{n+1}(t) \equiv d$ for $0 \leq t \leq T$.

Let $u^{l}=u^{l}(x, t)$ be the population density of the $l$ th species. For any $l \in\{1, \ldots, n\}$, the diffusion coefficients of the $l$ th species in $Q_{T}^{l-}, Q_{T}^{l+}$ are positive constants $\hat{a}^{l}$ and $\tilde{a}^{l}$, respectively. For any $l \in\{n+1, \ldots, N\}$, the diffusion coefficient of the $l$ th species in the whole 
domain $Q_{T}$ is positive constant $a^{l}$. Then Fick?s law shows that the flux of the population density can be represented as

$$
J^{l}= \begin{cases}-\hat{a}^{l} u_{x}^{l} & \left((x, t) \in Q_{T}^{l-}\right), l=1, \ldots, n, \\ -\tilde{a}^{l} u_{x}^{l} & \left((x, t) \in Q_{T}^{l+}\right), l=1, \ldots, n, \\ -a^{l} u_{x}^{l} & \left((x, t) \in Q_{T}\right), l=n+1, \ldots, N .\end{cases}
$$

Assume that for each $l \in\{1, \ldots, N\}$, harvesting rate or stocking rate for the $l$ th species depends on its density and flux. Using the principle of conservation, we conclude that the vector functions $\mathbf{u}=\left(u^{1}, \ldots, u^{N}\right)$ and $\boldsymbol{\varphi}=\left(\varphi^{1}, \ldots, \varphi^{n}\right)$ are governed by quasilinear reactiondiffusion equations

$$
\begin{aligned}
& u_{t}^{l}=\left\{\begin{array}{ll}
\hat{a}^{l} u_{x x}^{l}+\hat{b}^{l}\left(u^{l}\right) u_{x}^{l}+G^{l}(x, t, \mathbf{u}) & \left((x, t) \in Q_{T}^{l-}\right), \\
\tilde{a}^{l} u_{x x}^{l}+\tilde{b}^{l}\left(u^{l}\right) u_{x}^{l}+G^{l}(x, t, \mathbf{u}) & \left((x, t) \in Q_{T}^{l+}\right),
\end{array} \quad l=1, \ldots, n,\right. \\
& u_{t}^{l}=a^{l} u_{x x}^{l}+b^{l}\left(u^{l}\right) u_{x}^{l}+G^{l}(x, t, \mathbf{u}) \quad\left((x, t) \in Q_{T}\right), l=n+1, \ldots, N,
\end{aligned}
$$

where $\hat{b}^{i}\left(u^{i}\right) u_{x}^{i}, \tilde{b}^{i}\left(u^{i}\right) u_{x}^{i}(i=1, \ldots, n)$ and $b^{j}\left(u^{j}\right) u_{x}^{j}(j=n+1, \ldots, N)$ represent the mankind?s influence, and $G^{l}(x, t, \mathbf{u})(l=1, \ldots, N)$ are the reaction functions defined by (see the example in Section 5)

$$
G^{l}(x, t, \mathbf{u}):=g_{k}^{l}(\mathbf{u}) \quad\left((x, t) \in Q_{k, T}, k=1, \ldots, n+1\right)
$$

Here and below,

$$
u_{t}^{l}:=\partial u^{l} / \partial t, \quad u_{x}^{l}:=\partial u^{l} / \partial x, \quad u_{x x}^{l}:=\partial^{2} u^{l} / \partial x^{2} .
$$

On the parabolic boundary, $\mathbf{u}$ is required to satisfy the condition

$$
u^{l}=\psi^{l}(x, t) \quad\left((x, t) \in S_{T} \cup([0, d] \times\{0\})\right), l=1, \ldots, N .
$$

For each $l \in\{1, \ldots, n\}$, on the free boundary $\Gamma_{T}^{l}$, assume that the density and the flux are continuous (see [1]). By the conservation law of population, the rate of increase of population $\varphi_{t}^{l}(t) u^{l-}\left(\varphi^{l}(t), t\right)$ is equal to the flux $-\hat{a}^{l} u_{x}^{l-}\left(\varphi^{l}(t), t\right)$, where $u^{l-}\left(\varphi^{l}(t), t\right)$ and $u^{l+}\left(\varphi^{l}(t), t\right)$ represent the limits of $u^{l}$ from left and right for the space variable, respectively. Then $\mathbf{u}$ and $\varphi$ are required to satisfy the free boundary conditions

$$
\begin{aligned}
& \left\{\begin{array}{l}
u^{l-}\left(\varphi^{l}(t), t\right)=u^{l+}\left(\varphi^{l}(t), t\right), \\
\hat{a}^{l} u_{x}^{l-}\left(\varphi^{l}(t), t\right)=\tilde{a}^{l} u_{x}^{l+}\left(\varphi^{l}(t), t\right) \quad(t \in[0, T]), l=1, \ldots, n,
\end{array}\right. \\
& \varphi^{l}(0)=d^{l}, \quad \varphi_{t}^{l}(t) u^{l-}\left(\varphi^{l}(t), t\right)=-\hat{a}^{l} u_{x}^{l-}\left(\varphi^{l}(t), t\right) \quad(t \in[0, T]), l=1, \ldots, n .
\end{aligned}
$$

Condition (1.4) is used to describe the rates of change of the free boundaries.

The motivation to deal with problem (1.1a)-(1.4) are some real processes. For example, consider 4-species system with two predators and two preys, where two predator species comprise two groups of wolves and two groups of lions, two prey species comprise a herd of sheep and a herd of horses, and four species share the same habitat. In each of predator 
species, the two groups live in different habitats separated by a free boundary and have different diffusion coefficients and biological habits. Then this ecological system can be described by problem (1.1a)-(1.4).

The aim of this paper is to investigate: (i) the global existence and uniqueness of the solutions for diffraction problem (1.1a)-(1.3); (ii) the local existence of the solutions for problem (1.1a)-(1.4).

The free boundary problems often appear in different fields, such as physics, ecology and chemistry. They have been investigated extensively in the literature (see [1-13] and the references therein). For the free boundary problems of single equations, the linear problems describing the flow of fluids in a one-dimensional porous medium were studied by Kamynin [2] and Evans and Friedman [3-5], and the quasilinear problem was studied by Liang [6]. The work in [1] is concerned with the other quasilinear problem describing ecological model with one-species. For the free boundary problems of parabolic systems, Mimura et al. [7], Ling et al. [8] and Lin [9] investigated the problems arising from twospecies models in ecology, where the number of the free boundaries is one and the conditions on the free boundary are Stefan conditions. In this paper, there are $n$ free boundaries in problem (1.1a)-(1.4) and the vector functions $\mathbf{g}_{i}(\mathbf{u}):=\left(g_{i}^{1}(\mathbf{u}), \ldots, g_{i}^{N}(\mathbf{u})\right), i=1, \ldots, n$, are mixed quasimonotone.

Since for any given $\varphi(t)$, problem (1.1a)-(1.3) is a diffraction problem, we must first investigate the corresponding diffraction problem. In the study of diffraction problem, $\Gamma_{T}^{i}$, $i=1, \ldots, n$, are called inner boundaries. The papers in [14-16] are concerned with the diffraction problems of parabolic systems with the inner boundaries in the special form $\left\{x: \varphi_{k}(x)=0\right\} \times[0, T], k=1, \ldots, K$, where $\varphi_{k}(x) \in C^{2+\alpha_{0}}$. In this paper, by the approximation and estimate methods, we show the existence and uniqueness of the solutions for diffraction problem (1.1a)-(1.3) with the inner boundaries in the form $\Gamma_{T}^{i}: x=\varphi^{i}(t)$, $i=1, \ldots, n$, where $\varphi^{i}(t) \in W_{2}^{1}(0, T)$.

The rest of this paper is organized as follows. In Section 2, we state the notations, definitions, hypotheses and main results. Section 3 is devoted to investigating the global existence and uniqueness of the solutions for the corresponding diffraction problem (1.1a)(1.3). Section 4 is concerned with the local existence of solutions for the free boundary problem (1.1a)-(1.4) by Schauder?s fixed point theorem. Finally, in Section5 we give an example.

\section{The notations, hypotheses and main results}

Fist, let us define some basic function spaces (see [17])

$$
\begin{aligned}
& W_{p}^{1}(D):=\left\{v(x): v, v_{x} \in L^{p}(D)\right\} \quad(p \geq 1), \\
& W_{2}^{1,0}\left(Q_{T}\right):=\left\{u(x, t): u, u_{x} \in L^{2}\left(Q_{T}\right)\right\}, \\
& W_{2}^{1,1}\left(Q_{T}\right):=\left\{u(x, t): u, u_{x}, u_{t} \in L^{2}\left(Q_{T}\right)\right\},
\end{aligned}
$$

equipped with the norms

$$
\begin{aligned}
& \|v\|_{W_{p}^{1}(D)}:=\|v\|_{L^{p}(D)}+\left\|v_{x}\right\|_{L^{p}(D)}, \\
& \|u\|_{W_{2}^{1,0}\left(Q_{T}\right)}:=\|u\|_{L^{2}\left(Q_{T}\right)}+\left\|u_{x}\right\|_{L^{2}\left(Q_{T}\right)},
\end{aligned}
$$




$$
\|u\|_{W_{2}^{1,1}\left(Q_{T}\right)}:=\|u\|_{L^{2}\left(Q_{T}\right)}+\left\|u_{x}\right\|_{L^{2}\left(Q_{T}\right)}+\left\|u_{t}\right\|_{L^{2}\left(Q_{T}\right)}
$$

respectively, where $D \subset \mathbb{R}$ is an arbitrary interval.

$V_{2}\left(Q_{T}\right)$ is the Banach space consisting of all elements of $W_{2}^{1,0}\left(Q_{T}\right)$ having a finite norm

$$
\|v\|_{V_{2}\left(Q_{T}\right)}:=\underset{0 \leq t \leq T}{\operatorname{ess} \sup }\|v\|_{L^{2}(0, d)}+\left\|v_{x}\right\|_{L^{2}\left(Q_{T}\right)}
$$

$V_{2}^{1,0}\left(Q_{T}\right)$ is the Banach space consisting of all elements of $V_{2}\left(Q_{T}\right)$ that are continuous in $t$ in the norm of $L^{2}(0, d)$ with a finite norm

$$
\|v\|_{V_{2}^{1,0}\left(Q_{T}\right)}:=\max _{0 \leq t \leq T}\|v\|_{L^{2}(0, d)}+\left\|v_{x}\right\|_{L^{2}\left(Q_{T}\right)}
$$

$\stackrel{\circ}{W}_{2}^{1,1}\left(Q_{T}\right)$ is the set of all functions in $W_{2}^{1,1}\left(Q_{T}\right)$ that vanish on $S_{T}$ in the sense of trace. We write $H^{1}(0, d):=W_{2}^{1}(0, d)$.

$C^{\alpha}\left(\bar{Q}_{T}\right)$ is the spaces of Hölder continuous functions in $\bar{Q}_{T}$ with exponent $\alpha \in(0,1)$.

The product function spaces with $N$-components of $W_{2}^{1,1}\left(Q_{T}\right), V_{2}\left(Q_{T}\right), V_{2}^{1,0}\left(Q_{T}\right)$ and $C^{\alpha}\left(\bar{Q}_{T}\right)$ are denoted by $\mathcal{W}_{2}^{1,1}\left(\mathcal{Q}_{T}\right), \mathcal{V}_{2}\left(\mathcal{Q}_{T}\right), \mathcal{V}_{2}^{1,0}\left(\mathcal{Q}_{T}\right)$ and $\mathcal{C}^{\alpha}\left(\overline{\mathcal{Q}}_{T}\right)$, respectively, and the product function spaces with $n$-components of $W_{p}^{1}(0, T), L^{p}(0, T)$ and $C^{\alpha}([0, T])$ are denoted by $\mathbf{W}_{p}^{1}(0, T), \mathbf{L}^{p}(0, T)$ and $\mathbf{C}^{\alpha}([0, T])$, respectively. We note that the norm of $\boldsymbol{\varphi}(t)$ in $\mathbf{L}^{p}([0, T])$ is defined by

$$
\|\boldsymbol{\varphi}\|_{\mathbf{L}^{p}([0, T])}:=\left\|\varphi^{1}\right\|_{L^{p}([0, T])}+\cdots+\left\|\varphi^{n}\right\|_{L^{p}([0, T])}
$$

Let us recall the following definition. Write the vector $\mathbf{u}$ in the split form

$$
\mathbf{u}=\left(u^{l},[\mathbf{u}]_{\rho}^{l},[\mathbf{u}]_{\omega^{l}}\right),
$$

and rewrite the functions $f^{l}$ as

$$
f^{l}(x, t, \mathbf{u})=f^{l}\left(x, t, u^{l},[\mathbf{u}]_{\rho^{l}},[\mathbf{u}]_{\omega^{l}}\right), \quad l=1, \ldots, N,
$$

where $\rho^{l}, \omega^{l}$ are two nonnegative integers such that $\rho^{l}+\omega^{l}=N-1$ and $[\mathbf{u}]_{\sigma}$ denotes a vector with $\sigma$ number of component of $\mathbf{u}$. The split form of $\mathbf{u}$ varies with respect to $l$ and is determined by the quasimonotone property of $f^{l}$ given as follows.

Definition 2.1 (see [16]) The vector function $\mathbf{f}(\cdot, \mathbf{u}):=\left(f^{1}(\cdot, \mathbf{u}), \ldots, f^{N}(\cdot, \mathbf{u})\right)$ is said to be mixed quasimonotone in $\mathcal{J} \subset \mathbb{R}^{N}$ with index vector $\left([\mathbf{u}]_{\rho} ; \ldots ;[\mathbf{u}]_{\rho^{N}}\right)$ if for each $l=$ $1, \ldots, N$, there exist nonnegative integers $\varrho^{l}, \omega^{l}$, satisfying the relation

$$
\rho^{l}+\omega^{l}=N-1,
$$

such that $f^{l}\left(\cdot, u^{l},[\mathbf{u}]_{\rho^{l}},[\mathbf{u}]_{\omega^{l}}\right)$ is nondecreasing in $[\mathbf{u}]_{\varrho^{l}}$ and is nonincreasing in $[\mathbf{u}]_{\omega^{l}}$ for all $\mathbf{u} \in \mathcal{J}$.

Throughout the paper we make the following hypotheses: 
$(\mathrm{H})$

(i) $d^{i}, i=1, \ldots, n$, are constants satisfying the relation $0<d^{1}<\cdots<d^{n}<d . \psi^{l}(x, t)$,

$l=1, \ldots, N$, are all positive functions, and they possess the following properties: for some constants $\alpha_{0} \in(0,1)$ and $\chi_{0} \in\left(0, \min \left\{d^{1}, d-d^{n}\right\}\right)$,

$$
\begin{aligned}
& \psi^{l}(x, t) \in C^{\alpha_{0}}\left(\left[-\chi_{0}, d+\chi_{0}\right] \times[0, T]\right) \cap W_{2}^{1,1}\left(\left(-\chi_{0}, d+\chi_{0}\right) \times(0, T)\right), \\
& \psi^{l}(x, t) \in C^{1+\alpha_{0}}\left(\left[-\chi_{0}, \chi_{0}\right] \times[0, T]\right) \cap C^{1+\alpha_{0}}\left(\left[d-\chi_{0}, d+\chi_{0}\right] \times[0, T]\right), \\
& \quad l=1, \ldots, N \\
& \psi^{i}(x, 0) \in H^{1}(0, d) \cap C^{2+\alpha_{0}}\left(\left[0, d^{i}\right]\right) \cap C^{2+\alpha_{0}}\left(\left[d^{i}, d\right]\right), \quad i=1, \ldots, n, \\
& \psi^{j}(x, 0) \in C^{2+\alpha_{0}}([0, d]), \quad j=n+1, \ldots, N,
\end{aligned}
$$

and

$$
\hat{a}^{i} \psi_{x}^{i-}\left(d^{i}, 0\right)=\tilde{a}^{i} \psi_{x}^{i+}\left(d^{i}, 0\right), \quad i=1, \ldots, n
$$

(ii) There exist constant vectors $\mathbf{M}:=\left(M^{1}, \ldots, M^{N}\right)$ and $\mathbf{m}:=\left(m^{1}, \ldots, m^{N}\right), \mathbf{0}<\mathbf{m} \leq \mathbf{M}$, such that for each $l=1, \ldots, N$,

$$
\left\{\begin{array}{l}
g_{k}^{l}\left(M^{l},[\mathbf{M}]_{\varrho^{l}},[\mathbf{m}]_{\omega^{l}}\right) \leq 0, \\
g_{k}^{l}\left(m^{l},[\mathbf{m}]_{\varrho^{l}},[\mathbf{M}]_{\omega^{l}}\right) \geq 0, \quad k=1, \ldots, n+1, \\
m^{l}<\psi^{l}(x, t)<M^{l} \quad\left((x, t) \in S_{T} \cup\{\Omega \times\{0\}\}\right),
\end{array}\right.
$$

where the component $[\mathbf{u}]_{\varrho^{l}}$ is independent of $k$. Let

$$
\mathscr{S}:=\left\{\mathbf{u} \in \mathcal{C}\left(\overline{\mathcal{Q}}_{T}\right): \mathbf{m} \leq \mathbf{u} \leq \mathbf{M}\right\}
$$

The vector functions $\mathbf{g}_{k}(\mathbf{u})=\left(g_{k}^{1}(\mathbf{u}), \ldots, g_{k}^{N}(\mathbf{u})\right), k=1, \ldots, n+1$, are mixed quasimonotone in $\mathscr{S}$ with the same index vector $\left([\mathbf{u}]_{\rho^{1}} ; \ldots ;[\mathbf{u}]_{\rho^{N}}\right)$.

(iii) $\hat{b}^{i}\left(u^{i}\right), \tilde{b}^{i}\left(u^{i}\right), b^{j}\left(u^{j}\right) \in C^{1+\alpha_{0}}(\mathbb{R}), i=1, \ldots, n, j=n+1, \ldots, N$, and $g_{k}^{l}(\mathbf{u}) \in C^{1+\alpha_{0}}(\mathscr{S})$, $k=1, \ldots, n+1, l=1, \ldots, N$. There exists a positive nondecreasing function $\mu(\theta)$ for $\theta \in[0,+\infty)$ such that

$$
\left\{\begin{array}{l}
\left|\hat{b}^{i}\left(u^{i}\right), \tilde{b}^{i}\left(u^{i}\right)\right| \leq \mu\left(\left|u^{i}\right|\right), \quad i=1, \ldots, n \\
\left|b^{j}\left(u^{j}\right)\right| \leq \mu\left(\left|u^{j}\right|\right), \quad j=n+1, \ldots, N
\end{array}\right.
$$

Hypothesis (H)(i) implies that there exists a positive constant $\mu_{1}$ such that

$$
\begin{aligned}
& \left\|\psi^{l}(x, t)\right\|_{C^{\alpha_{0}}\left(\bar{Q}_{T}\right)}+\left\|\psi^{l}(x, t)\right\|_{W_{2}^{1,1}\left(Q_{T}\right)}+\left\|\psi^{l}(x, 0)\right\|_{H^{1}(0, d)} \\
& \quad+\left\|\psi_{x}^{l}(x, 0)\right\|_{C\left(\left[0, \delta_{0}\right]\right)}+\left\|\psi_{x}^{l}(x, 0)\right\|_{C\left(\left[d-\delta_{0}, d\right]\right)}+\left\|\psi^{l}(x, t)\right\|_{C^{1+\alpha_{0}\left(\left[0, \chi_{0}\right] \times[0, T]\right)}} \\
& \quad+\left\|\psi^{l}(x, t)\right\|_{C^{1+\alpha_{0}\left(\left[d-\chi_{0}, d\right] \times[0, T]\right)}} \leq \mu_{1}, \quad l=1, \ldots, N,
\end{aligned}
$$

where $\delta_{0}:=\min _{i=1, \ldots, n+1}\left(d^{i}-d^{i-1}\right), d^{0}=0$ and $d^{n+1}=d$. Hypothesis $(\mathrm{H})(\mathrm{ii})$ is used to guarantee the existence of the coupled weak upper and lower solutions for the approximation 
problem of (1.1a)-(1.3). (H)(iii) shows that on $Q_{T}$ the coefficients of the equations are allowed to be discontinuous, but on $Q_{k, T}$ the reaction functions are required to be independent of $(x, t)$ and have Hölder continuous derivatives with respect to $\mathbf{u}$, and on $Q_{T}^{l-}$ and $Q_{T}^{l_{+}}$ the remaining coefficients of the $l$ th equation are also required to be independent of $(x, t)$ and have Hölder continuous derivatives with respect to $\mathbf{u}$.

For convenience, let

$$
A^{l}(x, t):= \begin{cases}\hat{a}^{l} & \left((x, t) \in Q_{T}^{l-}\right), l=1, \ldots, n, \\ \tilde{a}^{l} & \left((x, t) \in Q_{T}^{l+}\right), l=1, \ldots, n, \\ a^{l} & \left((x, t) \in Q_{T}\right), l=n+1, \ldots, N,\end{cases}
$$

and

$$
B^{l}\left(x, t, u^{l}\right):= \begin{cases}\hat{b}^{l}\left(u^{l}\right) & \left((x, t) \in Q_{T}^{l-}\right), l=1, \ldots, n, \\ \tilde{b}^{l}\left(u^{l}\right) & \left((x, t) \in Q_{T}^{l+}\right), l=1, \ldots, n, \\ b^{l}\left(u^{l}\right) & \left((x, t) \in Q_{T}\right), l=n+1, \ldots, N .\end{cases}
$$

Definition 2.2 For a given $\varphi(t) \in \mathbf{W}_{2}^{1}(0, T)$, a vector function $\mathbf{u}(x, t)$ is said to be a solution of diffraction problem (1.1a)-(1.3) corresponding to $\varphi(t)$ if (i) For each $l=1, \ldots, N, u^{l} \in$ $C^{\alpha, \alpha / 2}\left(\bar{Q}_{T}\right)$ for some $0<\alpha<1, u^{l} \in V_{2}^{1,0}\left(Q_{T}\right), A^{l}(x, t) u_{x}^{l} \in V_{2}\left(Q_{T}\right), u_{t}^{l} \in L^{2}\left(Q_{T}\right)$, and $u^{l} \in$ $C^{2+\alpha, 1+\alpha / 2}\left(\bar{Q}^{\prime}\right)$ for any given $k \in\{1, \ldots, n+1\}$ and any given subdomain $Q^{\prime}, \bar{Q}^{\prime} \subset Q_{k, T}$; (ii) $\mathbf{u}$ satisfies pointwise the equations in (1.1a) and (1.1b) for $(x, t) \in Q_{k, T}(k=1, \ldots, n+1)$ and the parabolic boundary condition (1.2), and satisfies the inner boundary condition (1.3) for almost all $t \in[0, T]$; (iii) For any vector function $\eta=\left(\eta^{1}, \ldots, \eta^{N}\right) \in \mathcal{W}_{2}^{1,1}\left(\mathcal{Q}_{T}\right)$ and any $\tau \in[0, T]$, the following equalities hold:

$$
\begin{aligned}
& \left.\int_{0}^{d} u^{l} \eta^{l} \mathrm{~d} x\right|_{0} ^{\tau}+\iint_{Q_{\tau}}\left\{-u^{l} \eta_{t}^{l}+A^{l}(x, t) u_{x}^{l} \eta_{x}^{l}-\left[B^{l}\left(x, t, u^{l}\right) u_{x}^{l}+G^{l}(x, t, \mathbf{u})\right] \eta^{l}\right\} \mathrm{d} x \mathrm{~d} t \\
& \quad=0, \quad l=1, \ldots, N .
\end{aligned}
$$

Definition 2.3 $\{\mathbf{u}(x, t), \varphi(t)\}$ is said to be a solution of problem (1.1a)-(1.4) if (i) $\mathbf{u}$ is a solution of diffraction problem (1.1a)-(1.3) corresponding to $\varphi(t)$; (ii) $\varphi(t)$ is in $\mathbf{C}^{2+\alpha}((0, T])$, and (1.4) holds for $t \in(0, T]$.

Throughout the paper, $\alpha_{1}, \alpha_{2}, C(\cdots)$ and $C_{j}(\cdots)(j=1,2, \ldots)$ denote constants depending only on $T, d, d^{i}, 1 / m^{i}, M^{i}(i=1, \ldots, n), \mu(|\mathbf{M}|), \mu_{1}$, and the quantities appearing in parentheses. Constant $C$ in different expressions may be different.

The main results in this paper are the following theorems.

Theorem 2.1 Let hypothesis (H) be satisfied.

(i) If $\boldsymbol{\varphi}$ is in $\mathbf{W}_{2}^{1}(0, T)$ and satisfies

$$
\varphi(0)=\left(d^{1}, \ldots, d^{n}\right), \quad \min _{i=1, \ldots, n+1} \min _{t \in[0, T]}\left\{\varphi^{i}(t)-\varphi^{i-1}(t)\right\} \geq \delta>0
$$

for some $\delta>0$, then the corresponding diffraction problem (1.1a)-(1.3) has a unique solution $\mathbf{u}$ in $\mathscr{S}$. Moreover, the following estimates hold:

$$
\left\|u^{l}\right\|_{C^{\alpha_{1}, \alpha_{1} / 2}\left(\bar{Q}_{T}\right)} \leq C_{1} \quad\left(0<\alpha_{1}<1\right),
$$




$$
\begin{aligned}
& \left\|u^{l}\right\|_{V_{2}^{1,0}\left(Q_{T}\right)} \leq C_{2}(1 / \delta), \\
& \quad \underset{0 \leq t \leq T}{\operatorname{essup}}\left\|u_{x}^{l}\right\|_{L^{2}(0, d)}+\left\|u_{t}^{l}\right\|_{L^{2}\left(Q_{T}\right)}+\left\|\left(A(x, t) u_{x}^{l}\right)_{x}\right\|_{L^{2}\left(Q_{T}\right)} \\
& \quad \leq C_{3}\left(1 / \delta,\left\|\boldsymbol{\varphi}_{t}\right\|_{\mathbf{L}^{2}(0, T)}\right), \\
& \left\|u_{x}^{l}\right\|_{L^{\infty, 4}\left(Q_{T}\right)} \leq C_{4}\left(1 / \delta,\left\|\boldsymbol{\varphi}_{t}\right\|_{\mathbf{L}^{2}(0, T)}\right), \quad l=1, \ldots, N .
\end{aligned}
$$

(ii) If $\boldsymbol{\varphi}_{1}, \boldsymbol{\varphi}_{2}$ are in $\mathbf{W}_{2}^{1}(0, T)$ and satisfy (2.5), and if $\mathbf{u}_{1}, \mathbf{u}_{2}$ are the solutions in $\mathscr{S}$ corresponding to $\varphi_{1}$ and $\varphi_{2}$, respectively, then

$$
\begin{aligned}
\left\|u_{1}^{l}-u_{2}^{l}\right\|_{V_{2}^{1,0}\left(Q_{T}\right)}^{2} \leq C\left\|\boldsymbol{\varphi}_{1}-\boldsymbol{\varphi}_{2}\right\|_{\mathbf{L}^{2}(0, T)}, \\
\left\|u_{1}^{l}-u_{2}^{l}\right\|_{L^{\infty, 4}\left(Q_{T}\right)}^{2} \leq C\left\|\boldsymbol{\varphi}_{1}-\boldsymbol{\varphi}_{2}\right\|_{\mathbf{L}^{2}(0, T)}, \quad l=1, \ldots, N .
\end{aligned}
$$

(iii) If $\boldsymbol{\varphi}$ is in $\mathbf{C}^{2}((0, T])$ and $\mathbf{u}$ is the solution in $\mathscr{S}$, then for any given $t_{1} \in(0, T)$ and any given $l \in\{1, \ldots, n\}, u_{x}^{l}(x, t)$ and $u_{t}^{l}(x, t)$ are continuous with respect to $x$ in $\left[\varphi^{l}(t)-\delta / 4, \varphi^{l}(t)\right]$ and in $\left[\varphi^{l}(t), \varphi^{l}(t)+\delta / 4\right]$ for almost all $t \in\left[t_{1}, T\right]$, and $u_{x t}^{l}$ is square integrable in $Q^{l}\left(\delta, t_{1}\right)$, where $Q^{l}\left(\delta, t_{1}\right):=\left\{(x, t): x \in\left(\varphi^{l}(t)-\delta / 4, \varphi^{l}(t)+\delta / 4\right), t \in\left(t_{1}, T\right)\right\}$.

Theorem 2.2 Let hypothesis $(\mathrm{H})$ hold. Then there exist $T>0$ and a pair of vector functions $\mathbf{u}(x, t), \varphi(t)$, such that $\{\mathbf{u}(x, t), \varphi(t)\}$ is a solution to free boundary problem (1.1a)-(1.4).

\section{The corresponding diffraction problem}

Let $\varphi(t)$ be given and let (2.5) hold for some $\delta>0$. The aim of this section is to prove Theorem 2.1.

\subsection{An approximation problem}

We will construct an approximation problem of (1.1a)-(1.3). We first construct some approximation functions.

For an arbitrary $\varepsilon>0$, choose $\boldsymbol{\varphi}_{\varepsilon}=\left(\varphi_{\varepsilon}^{1}(t), \ldots, \varphi_{\varepsilon}^{n}(t)\right) \in \mathbf{C}^{2}([0, T])$ such that $\boldsymbol{\varphi}_{\varepsilon}(t) \rightarrow \boldsymbol{\varphi}(t)$ in $\mathbf{W}_{2}^{1}(0, T)$ as $\varepsilon \rightarrow 0$. Then, for small enough $\varepsilon$,

$$
\left\|\boldsymbol{\varphi}_{\varepsilon}\right\|_{\mathbf{W}_{2}^{1}(0, T)} \leq\|\boldsymbol{\varphi}\|_{\mathbf{W}_{2}^{1}(0, T)}+1
$$

Let $s_{\varepsilon}=s_{\varepsilon}(\theta)$ be a smooth function with values between 0 and 1 such that $\left|\mathrm{d} s_{\varepsilon}(\theta) / \mathrm{d} \theta\right| \leq$ $C / \varepsilon$ for all $\theta \in \mathbb{R}, s_{\varepsilon}(\theta)=1$ for $\theta \leq 0$ and $s_{\varepsilon}(\theta)=0$ for $\theta \geq \varepsilon$, and let

$$
z_{\varepsilon, k}(x, t):=\left\{\begin{array}{l}
\prod_{\tau=1}^{n} s_{\varepsilon}\left(x-\varphi_{\varepsilon}^{\tau}(t)\right) \quad\left((x, t) \in Q_{T}\right), k=1, \\
\prod_{\vartheta=1}^{n}\left[1-s_{\varepsilon}\left(x-\varphi_{\varepsilon}^{\vartheta}(t)\right)\right] \quad\left((x, t) \in Q_{T}\right), k=n+1, \\
\prod_{\tau=k}^{n} s_{\varepsilon}\left(x-\varphi_{\varepsilon}^{\tau}(t)\right) \prod_{\vartheta=1}^{k-1}\left[1-s_{\varepsilon}\left(x-\varphi_{\varepsilon}^{\vartheta}(t)\right)\right] \quad\left((x, t) \in Q_{T}\right), k=2, \ldots, n .
\end{array}\right.
$$

Define

$$
\begin{aligned}
& A_{\varepsilon}^{l}(x, t):=\left\{\begin{array}{l}
\hat{a}^{l} s_{\varepsilon}\left(x-\varphi_{\varepsilon}^{l}(t)\right)+\tilde{a}^{l}\left[1-s_{\varepsilon}\left(x-\varphi_{\varepsilon}^{l}(t)\right)\right] \quad\left((x, t) \in Q_{T}\right), l=1, \ldots, n, \\
a^{l} \quad\left((x, t) \in Q_{T}\right), l=n+1, \ldots, N,
\end{array}\right. \\
& B_{\varepsilon}^{l}\left(x, t, u^{l}\right):=\left\{\begin{array}{l}
\hat{b}^{l}\left(u^{l}\right) s_{\varepsilon}\left(x-\varphi_{\varepsilon}^{l}(t)\right)+\tilde{b}^{l}\left(u^{l}\right)\left[1-s_{\varepsilon}\left(x-\varphi_{\varepsilon}^{l}(t)\right)\right] \\
\quad\left((x, t) \in Q_{T}\right), l=1, \ldots, n, \\
b^{l}\left(u^{l}\right) \quad\left((x, t) \in Q_{T}\right), l=n+1, \ldots, N,
\end{array}\right.
\end{aligned}
$$


and

$$
G_{\varepsilon}^{l}(x, t, \mathbf{u}):=\sum_{k=1}^{n+1} g_{k}^{l}(\mathbf{u}) z_{\varepsilon, k}(x, t) \quad\left((x, t) \in Q_{T}\right), l=1, \ldots, N
$$

Then according to hypothesis $(\mathrm{H})(\mathrm{ii})$ and (iii), it follows that the vector function $\mathbf{G}_{\varepsilon}(\cdot, \mathbf{u})=$ $\left(G_{\varepsilon}^{1}(\cdot, \mathbf{u}), \ldots, G_{\varepsilon}^{N}(\cdot, \mathbf{u})\right)$ is mixed quasimonotone in $\mathscr{S}$ with index vector $\left([\mathbf{u}]_{\rho^{1}} ; \ldots ;[\mathbf{u}]_{\rho^{N}}\right)$, and

$$
\begin{aligned}
& A_{\varepsilon}^{l}(x, t) \in C^{1+\alpha_{0}}\left(\bar{Q}_{T}\right), \quad B_{\varepsilon}^{l}\left(x, t, u^{l}\right) \in C^{1+\alpha_{0}}\left(\bar{Q}_{T} \times \mathbb{R}\right), \\
& G_{\varepsilon}^{l}(x, t, \mathbf{u}) \in C^{1+\alpha_{0}}\left(\bar{Q}_{T} \times \mathscr{S}\right), \\
& v \leq A_{\varepsilon}^{l}(x, t) \leq C, \quad B_{\varepsilon}^{l}\left(x, t, u^{l}\right) \mid \leq \mu\left(\left|u^{l}\right|\right), \\
& \left|G_{\varepsilon}^{l}(x, t, \mathbf{u})\right| \leq C \quad\left((x, t) \in \bar{Q}_{T}, \mathbf{u} \in \mathscr{S}\right), \quad l=1, \ldots, N,
\end{aligned}
$$

where $v:=\min _{i=1, \ldots, n, j=n+1, \ldots, N}\left\{\hat{a}^{i}, \tilde{a}^{i}, a^{j}\right\}$. The definition of function $s_{\varepsilon}(\theta)$ implies that

$$
\begin{aligned}
& A_{\varepsilon}^{l}(x, t)= \begin{cases}\hat{a}^{l} & \left(x-\varphi_{\varepsilon}^{l}(t) \leq 0\right), \\
\tilde{a}^{l} & \left(x-\varphi_{\varepsilon}^{l}(t) \geq \varepsilon\right),\end{cases} \\
& B_{\varepsilon}^{l}\left(x, t, u^{l}\right)= \begin{cases}\hat{b}^{l}\left(u^{l}\right) & \left(x-\varphi_{\varepsilon}^{l}(t) \leq 0\right), \\
\tilde{b}^{l}\left(u^{l}\right) & \left(x-\varphi_{\varepsilon}^{l}(t) \geq \varepsilon\right),\end{cases}
\end{aligned}
$$

In addition, it is obvious from (2.5) that

$$
\bigcup_{\tau=1}^{k} \bar{Q}_{\tau, T}-\Gamma_{T}^{k}=\left\{(x, t): x-\varphi^{k}(t)<0\right\} \cap \bar{Q}_{T},
$$

where $\bar{Q}_{T}, \bar{Q}_{\tau, T}$ are the closure of $Q_{T}$ and $Q_{\tau, T}$, respectively. Thus by (3.9) and the definition of functions $z_{\varepsilon, k}(x, t)$, an argument similar to the one used in [16, Lemma 3.2] shows that

$$
\sum_{k=1}^{n+1} z_{\varepsilon, k}(x, t)=1 \quad\left((x, t) \in \bar{Q}_{T}\right)
$$

and

$$
G_{\varepsilon}^{l}(x, t, \mathbf{u})=\left\{\begin{array}{l}
g_{1}^{l}(\mathbf{u}) \quad \text { if } x-\varphi_{\varepsilon}^{1}(t) \leq 0 \\
g_{n+1}^{l}(\mathbf{u}) \quad \text { if } x-\varphi_{\varepsilon}^{n}(t) \geq \varepsilon \\
g_{k}^{l}(\mathbf{u}) \quad \text { if } x-\varphi_{\varepsilon}^{k-1}(t) \geq \varepsilon \text { and } x-\varphi_{\varepsilon}^{k}(t) \leq 0 \\
\quad \text { for some } k \in\{2, \ldots, n\}, \\
g_{k-1}^{l}(\mathbf{u}) s_{\varepsilon}\left(x-\varphi_{\varepsilon}^{k-1}(t)\right)+g_{k}^{l}(\mathbf{u})\left[1-s_{\varepsilon}\left(x-\varphi_{\varepsilon}^{k-1}(t)\right)\right] \\
\quad \text { if } 0<x-\varphi_{\varepsilon}^{k-1}(t)<\varepsilon \text { for some } k \in\{2, \ldots, n\} .
\end{array}\right.
$$

We next construct the approximation functions of $\psi^{l}(x, t)$. Let $\omega(|x|)$ be a sufficiently smooth nonnegative function such that $\omega(|x|)=0$ for $|x| \geq 1$ and $\int_{|x| \leq 1} \omega(x) \mathrm{d} x=1$, and let $\lambda=\lambda(x)$ be a sufficiently smooth nonnegative function taking values in $[0,1]$ such that $\lambda(x)=0$ for $\delta \leq x \leq d-\delta, \lambda(x)=1$ for $x \leq \delta / 2$ or $x \geq d-\delta / 2$, and $\lambda_{x}(x) \leq C / \delta$ for all $x \in \mathbb{R}$. 
Define

$$
\psi_{\varepsilon}^{l}=\psi_{\varepsilon}^{l}(x, t):=\int_{|x-y| \leq \varepsilon} \omega(|x-y|)(1-\lambda(y)) \psi^{l}(y, t) \mathrm{d} y+\lambda(x) \psi^{l}(x, t), \quad l=1, \ldots, N .
$$

Then hypothesis $(\mathrm{H})(\mathrm{i})$ and $[17$, Chapter II] imply that

$$
\left\{\begin{array}{l}
\psi_{\varepsilon}^{l}(x, t) \in C^{\alpha_{0}}\left(\bar{Q}_{T}\right) \cap W_{2}^{1,1}\left(Q_{T}\right), \quad \psi_{\varepsilon}^{l}(x, 0) \in C^{2+\alpha_{0}}([0, d]) \\
\psi_{\varepsilon}^{l}(0, t), \psi_{\varepsilon}^{l}(d, t) \in C^{2+\alpha_{0}}([0, T]), \quad l=1, \ldots, N
\end{array}\right.
$$

and

$$
\begin{aligned}
& \psi_{\varepsilon}^{l}(x, t) \rightarrow \psi^{l}(x, t) \quad \text { in } C^{\alpha_{0}}\left(\bar{Q}_{T}\right) \text { and in } W_{2}^{1,1}\left(Q_{T}\right), \\
& \psi_{\varepsilon}^{l}(x, 0) \rightarrow \psi^{l}(x, 0) \quad \text { in } H^{1}(0, d),
\end{aligned}
$$

and (2.1) and (2.3) imply that

$$
\begin{aligned}
& m^{l} \leq \psi_{\varepsilon}^{l}(x, t) \leq M^{l} \quad\left((x, t) \in S_{T} \cup\{\Omega \times\{0\}\}\right), \\
& \left\|\psi_{\varepsilon}^{l}(x, t)\right\|_{C^{\alpha_{0}}\left(\bar{Q}_{T}\right)} \leq \mu_{1}+1, \\
& \left\|\psi_{\varepsilon}^{l}(x, t)\right\|_{W_{2}^{1,1}\left(Q_{T}\right)}+\left\|\psi_{\varepsilon}^{l}(x, 0)\right\|_{H^{1}(0, d)}+\left\|\psi_{\varepsilon x}^{l}(x, 0)\right\|_{C\left(\left[0, \delta_{0}\right]\right)} \\
& \quad+\left\|\psi_{\varepsilon x}^{l}(x, 0)\right\|_{C\left(\left[d-\delta_{0}, d\right]\right)} \leq C(1 / \delta), \quad l=1, \ldots, N .
\end{aligned}
$$

In addition, for $\varepsilon<\delta / 8$,

$$
\psi_{\varepsilon}^{l}(x, t)=\psi^{l}(x, t) \quad((x, t) \in\{[0, \delta / 4] \cup[d-\delta / 4]\} \times[0, T]) .
$$

Employing the above approximation functions, we consider the following approximation problem:

$$
\left\{\begin{array}{l}
u_{t}^{l}-\left(A_{\varepsilon}^{l}(x, t) u_{x}^{l}\right)_{x}=B_{\varepsilon}^{l}\left(x, t, u^{l}\right) u_{x}^{l}+G_{\varepsilon}^{l}(x, t, \mathbf{u}) \quad\left((x, t) \in Q_{T}\right), \\
u^{l}=\psi_{\varepsilon}^{l}(x, t) \quad\left((x, t) \in S_{T} \cup([0, d] \times\{0\})\right), l=1, \ldots, N .
\end{array}\right.
$$

Lemma 3.1 Problem (3.16) has a unique classical solution $\mathbf{u}_{\varepsilon}$ in $\mathscr{S}$, and the following estimates hold:

$$
\begin{aligned}
& \left\|u_{\varepsilon}^{l}\right\|_{C^{\alpha_{2}, \alpha_{2} / 2}\left(\bar{Q}_{T}\right)} \leq C \quad\left(\alpha_{2} \in(0,1)\right), \\
& \left\|u_{\varepsilon}^{l}\right\|_{V_{2}^{1,0}\left(Q_{T}\right)} \leq C(1 / \delta), \\
& \left|u_{x}^{l}(0, t)\right|+\left|u_{x}^{l}(d, t)\right| \leq C(1 / \delta) \quad(t \in[0, T]), \\
& \max _{[0, T]}\left\|u_{\varepsilon x}^{l}\right\|_{L^{2}(0, d)}+\left\|u_{\varepsilon t}^{l}\right\|_{L^{2}\left(Q_{T}\right)}+\left\|\left(A_{\varepsilon}^{l}(x, t) u_{\varepsilon x}^{l}\right)_{x}\right\|_{L^{2}\left(Q_{T}\right)} \leq C\left(1 / \delta,\left\|\varphi_{t}\right\|_{L^{2}(0, T)}\right), \\
& \left\|u_{\varepsilon x}^{l}\right\|_{L^{\infty, 4}\left(Q_{T}\right)} \leq C\left(1 / \delta,\left\|\varphi_{t}\right\|_{L^{2}(0, T)}\right), \quad l=1, \ldots, N,
\end{aligned}
$$

where constants $\alpha_{2}$ and $C$ are independent of $\varepsilon$.

Proof In [15], by using the method of upper and lower solutions, together with the associated monotone iterations and various estimates, we investigated the existence and 
uniqueness of the global piecewise classical solutions of the quasilinear parabolic system with discontinuous coefficients and continuous delays under various conditions including mixed quasimonotone property of reaction functions. The same problem was also discussed for the system with continuous coefficients without time-delay.

It is obvious that problem (3.16) is the special case of [15, problem (1.1)] without discontinuous coefficients and time delays. Hypothesis (H)(ii) shows that $\tilde{\mathbf{u}}=\mathbf{M}, \hat{\mathbf{u}}=\mathbf{m}$ are the coupled weak upper and lower solutions of (3.16) in the sense of [15, Definition 2.2]. By (3.4)-(3.6) and (3.11)-(3.14), we conclude from [15, Theorem 4.1] that problem (3.16) has a unique classical solution $\mathbf{u}_{\varepsilon}$ in $\mathscr{S}$. Furthermore, using (3.1), (3.5), (3.6) and (3.12)-(3.14), the proof similar to that of [16, Lemma 3.3] shows that estimates (3.17) and (3.18) hold.

To prove (3.19), we first fix $l \in\{1, \ldots, n\}$. In view of (3.7), (3.8), (3.10) and (3.15), we find that $u_{\varepsilon}^{l}$ is the solution of the following problems for single equation:

$$
\left\{\begin{array}{l}
u_{\varepsilon t}^{l}-\left(\hat{a}^{l} u_{\varepsilon x}^{l}\right)_{x}=\hat{b}^{l}\left(u_{\varepsilon}^{l}\right) u_{\varepsilon x}^{l}+g_{1}^{l}\left(u_{\varepsilon}^{l},\left[\mathbf{u}_{\varepsilon}\right]_{\rho^{l}},\left[\mathbf{u}_{\varepsilon}\right]_{\omega^{l}}\right) \quad((x, t) \in(0, \delta / 4) \times(0, T]), \\
u_{\varepsilon}^{l}=\psi^{l}(x, t) \quad(x=0,0 \leq t \leq T \text { or } 0 \leq x \leq \delta / 4, t=0),
\end{array}\right.
$$

and

$$
\left\{\begin{array}{l}
u_{\varepsilon t}^{l}-\left(\tilde{a}^{l} u_{\varepsilon x}^{l}\right)_{x}=\tilde{b}^{l}\left(u_{\varepsilon}^{l}\right) u_{\varepsilon x}^{l}+g_{n+1}^{l}\left(u_{\varepsilon}^{l},\left[\mathbf{u}_{\varepsilon}\right]_{\rho} l,\left[\mathbf{u}_{\varepsilon}\right]_{\omega}\right) \\
\quad((x, t) \in(d-\delta / 4, d) \times(0, T]), \\
u_{\varepsilon}^{l}=\psi^{l}(x, t) \quad(x=d, 0 \leq t \leq T \text { or } d-\delta / 4 \leq x \leq d, t=0) .
\end{array}\right.
$$

By (3.22), (3.23), (3.12) and (3.14), the proof similar to that of [17, Chapter VI, Lemma 3.1] gives (3.19) for $l \in\{1, \ldots, n\}$. The similar argument shows that (3.19) holds for $l \in\{n+$ $1, \ldots, N\}$.

We next prove (3.20). For any fixed $l \in\{1, \ldots, n\}$, let

$$
y=x-\varphi_{\varepsilon}^{l}(t), \quad t^{\prime}=t \quad(\text { denoted by } t \text { still })
$$

and let

$$
q(y, t)=u_{\varepsilon}^{l}\left(y+\varphi_{\varepsilon}^{l}(t), t\right) .
$$

By a direct computation we have

$$
\left\{\begin{array}{l}
q_{y}(y, t)=u_{\varepsilon x}^{l}(x, t), \quad q_{t}(y, t)=q_{y}(y, t) \varphi_{\varepsilon t}^{l}+u_{\varepsilon t}^{l} \\
q_{y}\left(d-\varphi_{\varepsilon}^{l}(t), t\right)=u_{\varepsilon x}^{l}(d, t), \quad q_{y}\left(-\varphi_{\varepsilon}^{l}(t), t\right)=u_{\varepsilon x}^{l}(0, t) \\
q_{t}\left(d-\varphi_{\varepsilon}^{l}(t), t\right)=u_{\varepsilon x}^{l}(d, t) \varphi_{\varepsilon t}^{l}+\psi_{\varepsilon t}^{l}(d, t) \\
q_{t}\left(-\varphi_{\varepsilon}^{l}(t), t\right)=u_{\varepsilon x}^{l}(0, t) \varphi_{\varepsilon t}^{l}+\psi_{\varepsilon t}^{l}(0, t)
\end{array}\right.
$$

Then (3.16), (3.2), (3.3) and (3.10) imply that the function $q=q(y, t)$ satisfies

$$
\left\{\begin{aligned}
q_{t}-\left\{\left[\hat{a}^{l} s_{\varepsilon}(y)+\tilde{a}^{l}\left(1-s_{\varepsilon}(y)\right)\right] q_{y}\right\} & -q_{y} \varphi_{\varepsilon t}^{l} \\
& =B_{\varepsilon}^{l}\left(y+\varphi_{\varepsilon}^{l}(t), t, q\right) q_{y}+G_{\varepsilon}^{l}\left(y+\varphi_{\varepsilon}^{l}(t), t, \mathbf{u}_{\varepsilon}\left(y+\varphi_{\varepsilon}^{l}(t), t\right)\right) \quad\left((y, t) \in E_{T}^{l}\right), \\
q\left(-\varphi_{\varepsilon}^{l}(t), t\right)=\psi_{\varepsilon}^{l}(0, t), & q\left(d-\varphi_{\varepsilon}^{l}(t), t\right)=\psi_{\varepsilon}^{l}(d, t) \quad(t \in[0, T]), \\
q(y, 0)=\psi_{\varepsilon}^{l}\left(y+\varphi_{\varepsilon}^{l}(0), 0\right) & \left(y \in\left[-\varphi_{\varepsilon}^{l}(0), d-\varphi_{\varepsilon}^{l}(0)\right]\right),
\end{aligned}\right.
$$

where $E_{T}^{l}:=\left\{(y, t):-\varphi_{\varepsilon}^{l}(t)<y<d-\varphi_{\varepsilon}^{l}(t), 0<t \leq T\right\}$. 
A double integration by parts gives

$$
\begin{aligned}
-\iint_{E_{\tau}^{l}}\left\{\left[\hat{a}^{l} s_{\varepsilon}(y)+\tilde{a}^{l}\left(1-s_{\varepsilon}(y)\right)\right] q_{y}\right\}_{y} q_{t} \mathrm{~d} y \mathrm{~d} t \\
=\left.\frac{1}{2} \int_{-\varphi_{\varepsilon}^{l}(t)}^{d-\varphi_{\varepsilon}^{l}(t)}\left[\hat{a}^{l} s_{\varepsilon}(y)+\tilde{a}^{l}\left(1-s_{\varepsilon}(y)\right)\right]\left(q_{y}\right)^{2} \mathrm{~d} y\right|_{t=0} ^{t=\tau} \\
\quad+\left.\int_{0}^{\tau}\left[\hat{a}^{l} s_{\varepsilon}(y)+\tilde{a}^{l}\left(1-s_{\varepsilon}(y)\right)\right]\left\{-q_{y} q_{t}+\frac{1}{2} \varphi_{\varepsilon t}^{l}\left(q_{y}\right)^{2}\right\}\right|_{y=-\varphi_{\varepsilon}^{l}} ^{y=d-\varphi_{\varepsilon}^{l}} \mathrm{~d} t .
\end{aligned}
$$

Thus multiplying the equation in (3.25) by $q_{t}$, integrating it on $E_{\tau}^{l}$ and using (3.5), (3.6), (3.24) and (3.26), we find that

$$
\begin{aligned}
& \frac{1}{2} \int_{-\varphi_{\varepsilon}^{l}(\tau)}^{d-\varphi_{\varepsilon}^{l}(\tau)}\left[\hat{a}^{l} s_{\varepsilon}(y)+\tilde{a}^{l}\left(1-s_{\varepsilon}(y)\right)\right]\left(q_{y}(y, \tau)\right)^{2} \mathrm{~d} y+\iint_{E_{\tau}^{l}}\left(q_{t}\right)^{2} \mathrm{~d} y \mathrm{~d} t \\
& \leq C \int_{0}^{d}\left(\psi_{x}^{l}(x, 0)\right)^{2} \mathrm{~d} x+C \iint_{E_{\tau}^{l}}\left(\left|\varphi_{\varepsilon t}^{l}\right|+1\right)\left(\left|q_{y}\right|+1\right)\left|q_{t}\right| \mathrm{d} x \mathrm{~d} t \\
& \quad+C \int_{0}^{\tau}\left\{\left|u_{\varepsilon x}^{l}(0, t)\right|\left[\left|u_{\varepsilon x}^{l}(0, t) \varphi_{\varepsilon t}^{l}\right|+\left|\psi_{\varepsilon t}^{l}\right|\right]+\left|u_{\varepsilon x}^{l}(d, t)\right|\left[\left|u_{\varepsilon x}^{l}(d, t) \varphi_{\varepsilon t}^{l}\right|+\left|\psi_{\varepsilon t}^{l}\right|\right]\right. \\
& \left.\quad+\left|\varphi_{\varepsilon t}^{l}\right|\left[\left(u_{\varepsilon x}^{l}(0, t)\right)^{2}+\left(u_{\varepsilon x}^{l}(d, t)\right)^{2}\right]\right\} \mathrm{d} t .
\end{aligned}
$$

Furthermore, by (3.12)-(3.14), (3.19) and Cauchy?s inequality, we deduce that for anyo > 0,

$$
\begin{aligned}
& \frac{1}{2} v \int_{-\varphi_{\varepsilon}^{l}(\tau)}^{d-\varphi_{\varepsilon}^{l}(\tau)}\left(q_{y}(y, \tau)\right)^{2} \mathrm{~d} y+\iint_{E_{\tau}^{l}}\left(q_{t}\right)^{2} \mathrm{~d} y \mathrm{~d} t \\
& \quad \leq \sigma \iint_{E_{\tau}^{l}}\left(q_{t}\right)^{2} \mathrm{~d} y \mathrm{~d} t+C(\sigma) \iint_{E_{\tau}^{l}}\left[1+\left(q_{y}\right)^{2}\right]\left[1+\left(\varphi_{\varepsilon t}^{l}\right)^{2}\right] \mathrm{d} y \mathrm{~d} t+C\left(1 / \delta,\left\|\boldsymbol{\varphi}_{t}\right\|_{\mathbf{L}^{2}(0, T)}\right) .
\end{aligned}
$$

Choosing $\sigma=v / 4$, we get

$$
\begin{aligned}
& \int_{-\varphi_{\varepsilon}^{l}(\tau)}^{d-\varphi_{\varepsilon}^{l}(\tau)}\left(q_{y}(y, \tau)\right)^{2} \mathrm{~d} y+\iint_{E_{\tau}^{l}}\left(q_{t}\right)^{2} \mathrm{~d} y \mathrm{~d} t \\
& \quad \leq \int_{0}^{\tau}\left\{\left[1+\left(\varphi_{\varepsilon t}^{l}\right)^{2}\right] \int_{-\varphi_{\varepsilon}^{l}(t)}^{d-\varphi_{\varepsilon}^{l}(t)}\left(q_{y}(y, t)\right)^{2} \mathrm{~d} y\right\} \mathrm{d} t+C\left(1 / \delta,\left\|\boldsymbol{\varphi}_{t}\right\|_{\mathbf{L}^{2}(0, T)}\right) .
\end{aligned}
$$

Consequently,

$$
\begin{aligned}
{[1+} & \left.\left(\varphi_{\varepsilon t}^{l}(\tau)\right)^{2}\right] \int_{-\varphi_{\varepsilon}^{l}(\tau)}^{d-\varphi_{\varepsilon}^{l}(\tau)}\left(q_{y}(y, \tau)\right)^{2} \mathrm{~d} y \\
\leq & {\left[1+\left(\varphi_{\varepsilon t}^{l}(\tau)\right)^{2}\right] \int_{0}^{\tau}\left\{\left[1+\left(\varphi_{\varepsilon t}^{l}\right)^{2}\right] \int_{-\varphi_{\varepsilon}^{l}(t)}^{d-\varphi_{\varepsilon}^{l}(t)}\left(q_{y}(y, t)\right)^{2} \mathrm{~d} y\right\} \mathrm{d} t } \\
& +\left[1+\left(\varphi_{\varepsilon t}^{l}(\tau)\right)^{2}\right] C\left(1 / \delta,\left\|\boldsymbol{\varphi}_{t}\right\|_{\mathbf{L}^{2}(0, T)}\right) .
\end{aligned}
$$

This, together with Gronwall?s inequality (see 17, Chapter II, Lemma 5.5]), implies that

$$
\iint_{E_{\tau}^{l}}\left[1+\left(\varphi_{\varepsilon t}^{l}\right)^{2}\right]\left[1+\left(q_{y}\right)^{2}\right] \mathrm{d} y \mathrm{~d} t \leq C\left(1 / \delta,\left\|\boldsymbol{\varphi}_{t}\right\|_{\mathbf{L}^{2}(0, T)}\right)
$$


Hence we deduce from (3.25), (3.27), (3.5) and (3.6) that

$$
\begin{aligned}
& \max _{0 \leq t \leq T} \int_{-\varphi_{\varepsilon}^{l}(t)}^{d-\varphi_{\varepsilon}^{l}(t)}\left(q_{y}\right)^{2} \mathrm{~d} y+\iint_{E_{T}^{l}}\left\{\left(q_{t}\right)^{2}+\left[\left(\left(\hat{a}^{l} s_{\varepsilon}(y)+\tilde{a}^{l}\left(1-s_{\varepsilon}(y)\right)\right) q_{y}\right)_{y}\right]^{2}\right\} \mathrm{d} y \mathrm{~d} t \\
& \quad \leq C\left(1 / \delta,\left\|\boldsymbol{\varphi}_{t}\right\|_{\mathbf{L}^{2}(0, T)}\right),
\end{aligned}
$$

which, together with (3.24), yields (3.20) for $l \in\{1, \ldots, n\}$.

For any fixed $l \in\{n+1, \ldots, N\}$, we consider the equality

$$
\iint_{Q_{\tau}}\left\{u_{\varepsilon t}^{l}-\left(A_{\varepsilon}^{l}(x, t) u_{\varepsilon x}^{l}\right)_{x}-B_{\varepsilon}^{l}\left(x, t, u_{\varepsilon}^{l}\right) u_{\varepsilon x}^{l}-G_{\varepsilon}^{l}\left(x, t, \mathbf{u}_{\varepsilon}\right)\right\} u_{\varepsilon t}^{l} \mathrm{~d} x \mathrm{~d} t=0 .
$$

A similar argument gives (3.20) for $l \in\{n+1, \ldots, N\}$. Therefore, (3.20) holds for all $l \in$ $\{1, \ldots, N\}$.

It remains to prove (3.21). For each $l \in\{1, \ldots, N\}$, since $A_{\varepsilon}^{l}(x, t) u_{\varepsilon x}^{l}$ is in $V_{2}\left(Q_{T}\right)$, then (3.20) and [17, Chapter 2, formula (3.8)] show that

$$
\begin{aligned}
v\left\|u_{\varepsilon x}^{l}\right\|_{L^{\infty, 4}\left(Q_{T}\right)} & \leq\left\|A_{\varepsilon}^{l}(x, t) u_{\varepsilon x}^{l}\right\|_{L^{\infty, 4}\left(Q_{T}\right)} \\
& \leq\left\|\left(A_{\varepsilon}^{l}(x, t) u_{\varepsilon x}^{l}\right)_{x}\right\|_{L^{2}\left(Q_{T}\right)}+\sup _{0 \leq t \leq T}\left\|A_{\varepsilon}^{l}(x, t) u_{\varepsilon x}^{l}\right\|_{L^{2}(0, d)} \\
& \leq C\left(1 / \delta,\left\|\boldsymbol{\varphi}_{t}\right\|_{\mathbf{L}^{2}(0, T)}\right) .
\end{aligned}
$$

Thus (3.21) holds.

Lemma 3.2 Let $\mathbf{u}_{\varepsilon_{1}}(x, t), \mathbf{u}_{\varepsilon_{2}}(x, t)$ be the solutions in $\mathscr{S}$ for problem (3.16) corresponding to $\boldsymbol{\varphi}_{\varepsilon_{1}}(t)$ and $\boldsymbol{\varphi}_{\varepsilon_{2}}(t)$, respectively. Then

$$
\left\|\mathbf{u}_{\varepsilon_{1}}-\mathbf{u}_{\varepsilon_{2}}\right\|_{\mathcal{V}_{2}^{1,0}\left(\mathcal{Q}_{T}\right)}^{2} \leq C\left(1 / \delta,\left\|\boldsymbol{\varphi}_{t}\right\|_{\mathbf{L}^{2}(0, T)}\right) I_{3,0}
$$

where $I_{3,0}:=\sum_{l=1}^{N}\left\|\psi_{\varepsilon_{1}}^{l}(x, 0)-\psi_{\varepsilon_{2}}^{l}(x, 0)\right\|_{H^{1}(0, d)}^{2}+\left\|\boldsymbol{\varphi}_{\varepsilon_{1}}-\boldsymbol{\varphi}_{\varepsilon_{2}}\right\|_{\mathbf{L}^{2}(0, T)}+\varepsilon_{1}+\varepsilon_{2}$.

Proof Let $\mathbf{w}=\left(w^{1}, \ldots, w^{N}\right)=\mathbf{u}_{\varepsilon_{1}}-\mathbf{u}_{\varepsilon_{2}}$, and let $l \in\{1, \ldots, N\}$ be fixed. We see from (3.16) that

$$
\left\{\begin{aligned}
w_{t}^{l}= & \left\{\left[A_{\varepsilon_{1}}^{l}(x, t)-A_{\varepsilon_{2}}^{l}(x, t)\right] u_{\varepsilon_{1} x}^{l}\right\}_{x}+\left\{A_{\varepsilon_{2}}^{l}(x, t) w_{x}^{l}\right\}_{x} \\
& +\left[B_{\varepsilon_{1}}^{l}\left(x, t, u_{\varepsilon_{1}}^{l}\right) u_{\varepsilon_{1} x}^{l}-B_{\varepsilon_{2}}^{l}\left(x, t, u_{\varepsilon_{2}}^{l}\right) u_{\varepsilon_{2} x}^{l}\right] \\
& +\left[G_{\varepsilon_{1}}^{l}\left(x, t, \mathbf{u}_{\varepsilon_{1}}\right)-G_{\varepsilon_{2}}^{l}\left(x, t, \mathbf{u}_{\varepsilon_{2}}\right)\right] \quad\left((x, t) \in Q_{T}\right), \\
w^{l}= & \psi_{\varepsilon_{1}}^{l}(x, t)-\psi_{\varepsilon_{2}}^{l}(x, t) \quad\left((x, t) \in S_{T} \cup([0, d] \times\{0\})\right) .
\end{aligned}\right.
$$

In view of (3.15), we find $w^{l}(0, t)=w^{l}(d, t)=0$. Multiplying the equation in (3.29) by $w^{l}$ and integrating by parts on $Q_{\tau}$, we deduce that, for any $\tau \in[0, T]$,

$$
\begin{array}{r}
\left.\frac{1}{2} \int_{0}^{d}\left(w^{l}\right)^{2} \mathrm{~d} x\right|_{t=0} ^{t=\tau}+\iint_{Q_{\tau}} A_{\varepsilon_{2}}^{l}(x, t)\left(w_{x}^{l}\right)^{2} \mathrm{~d} x \mathrm{~d} t \\
\quad=\iint_{Q_{\tau}}-\left[A_{\varepsilon_{1}}^{l}(x, t)-A_{\varepsilon_{2}}^{l}(x, t)\right] u_{\varepsilon_{1} x}^{l} w_{x}^{l} \mathrm{~d} x \mathrm{~d} t
\end{array}
$$




$$
\begin{aligned}
& \quad+\iint_{Q_{\tau}}\left[B_{\varepsilon_{1}}^{l}\left(x, t, u_{\varepsilon_{1}}^{l}\right) u_{\varepsilon_{1} x}^{l}-B_{\varepsilon_{2}}^{l}\left(x, t, u_{\varepsilon_{2}}^{l}\right) u_{\varepsilon_{2} x}^{l}\right] w^{l} \mathrm{~d} x \mathrm{~d} t \\
& \quad+\iint_{Q_{\tau}}\left[G_{\varepsilon_{1}}^{l}\left(x, t, \mathbf{u}_{\varepsilon_{1}}\right)-G_{\varepsilon_{2}}^{l}\left(x, t, \mathbf{u}_{\varepsilon_{2}}\right)\right] w^{l} \mathrm{~d} x \mathrm{~d} t \\
& =: I_{3,1}^{l}+I_{3,2}^{l}+I_{3,3}^{l} .
\end{aligned}
$$

Let us estimate $I_{3,1}^{l}, I_{3,2}^{l}$ and $I_{3,3}^{l}$. Since (3.2), (3.3) and (3.10) imply that

$$
\begin{aligned}
& A_{\varepsilon_{1}}^{l}(x, t)-A_{\varepsilon_{2}}^{l}(x, t)=\left\{\begin{array}{l}
{\left[s_{\varepsilon_{1}}\left(x-\varphi_{\varepsilon_{1}}^{l}(t)\right)-s_{\varepsilon_{2}}\left(x-\varphi_{\varepsilon_{2}}^{l}(t)\right)\right]\left(\hat{a}^{l}-\tilde{a}^{l}\right), \quad l=1, \ldots, n,} \\
0, \quad l=n+1, \ldots, N,
\end{array}\right. \\
& \left|B_{\varepsilon_{1}}^{l}\left(x, t, u_{\varepsilon_{1}}^{l}\right) u_{\varepsilon_{1} x}^{l}-B_{\varepsilon_{2}}^{l}\left(x, t, u_{\varepsilon_{2}}^{l}\right) u_{\varepsilon_{2} x}^{l}\right| \\
& =\left\{\begin{array}{l}
\mid\left[s_{\varepsilon_{1}}\left(x-\varphi_{\varepsilon_{1}}^{l}(t)\right)-s_{\varepsilon_{2}}\left(x-\varphi_{\varepsilon_{2}}^{l}(t)\right)\right]\left[\hat{b}^{l}\left(u_{\varepsilon_{1}}^{l}\right)-\tilde{b}^{l}\left(u_{\varepsilon_{1}}^{l}\right)\right] u_{\varepsilon_{1} x}^{l} \\
\quad+s_{\varepsilon_{2}}\left(x-\varphi_{\varepsilon_{2}}^{l}(t)\right)\left[\hat{b}^{l}\left(u_{\varepsilon_{1}}^{l}\right) u_{\varepsilon_{1} x}^{l}-\hat{b}^{l}\left(u_{\varepsilon_{2}}^{l}\right) u_{\varepsilon_{2} x}^{l}\right] \\
\quad+\left[1-s_{\varepsilon_{2}}\left(x-\varphi_{\varepsilon_{2}}^{l}(t)\right)\right]\left[\tilde{b}^{l}\left(u_{\varepsilon_{1}}^{l}\right) u_{\varepsilon_{1} x}^{l}-\tilde{b}^{l}\left(u_{\varepsilon_{2}}^{l}\right) u_{\varepsilon_{2} x}^{l}\right] \mid, \quad l=1, \ldots, n, \\
\left|\left[b^{l}\left(u_{\varepsilon_{1}}^{l}\right)-b^{l}\left(u_{\varepsilon_{2}}^{l}\right)\right] u_{\varepsilon_{1} x}^{l}+b^{l}\left(u_{\varepsilon_{2}}^{l}\right)\left(u_{\varepsilon_{1} x}^{l}-u_{\varepsilon_{2} x}^{l}\right)\right|, \quad l=n+1, \ldots, N
\end{array}\right. \\
& \leq C \sum_{l=1}^{n}\left|\left[s_{\varepsilon_{1}}\left(x-\varphi_{\varepsilon_{1}}^{l}(t)\right)-s_{\varepsilon_{2}}\left(x-\varphi_{\varepsilon_{2}}^{l}(t)\right)\right]\right|\left|u_{\varepsilon_{1} x}^{l}\right|+C \sum_{l=1}^{N}\left[\left|w^{l}\right|\left|u_{\varepsilon_{1} x}^{l}\right|+\left|w_{x}^{l}\right|\right],
\end{aligned}
$$

and

$$
\begin{aligned}
G_{\varepsilon_{1}}^{l} & \left(x, t, \mathbf{u}_{\varepsilon_{1}}\right)-G_{\varepsilon_{2}}^{l}\left(x, t, \mathbf{u}_{\varepsilon_{2}}\right) \\
= & \left\{\begin{array}{l}
g_{1}^{l}\left(\mathbf{u}_{\varepsilon_{1}}\right)-g_{1}^{l}\left(\mathbf{u}_{\varepsilon_{2}}\right) \quad \text { if } x \leq \min \left(\varphi_{\varepsilon_{1}}^{1}(t), \varphi_{\varepsilon_{2}}^{1}(t)\right), \\
g_{n+1}^{l}\left(\mathbf{u}_{\varepsilon_{1}}\right)-g_{n+1}^{l}\left(\mathbf{u}_{\varepsilon_{2}}\right) \quad \text { if } x \geq \max \left(\varphi_{\varepsilon_{1}}^{n}(t)+\varepsilon_{1}, \varphi_{\varepsilon_{2}}^{n}(t)+\varepsilon_{2}\right), \\
g_{k}^{l}\left(\mathbf{u}_{\varepsilon_{1}}\right)-g_{k}^{l}\left(\mathbf{u}_{\varepsilon_{2}}\right) \quad \text { if } x \leq \min \left(\varphi_{\varepsilon_{1}}^{k}(t), \varphi_{\varepsilon_{2}}^{k}(t)\right) \text { and } \\
\quad x \geq \max \left(\varphi_{\varepsilon_{1}}^{k-1}(t)+\varepsilon_{1}, \varphi_{\varepsilon_{2}}^{k-1}(t)+\varepsilon_{2}\right) \text { for } k \in\{2, \ldots, n\}, \\
G_{\varepsilon_{1}}^{l}\left(x, t, \mathbf{u}_{\varepsilon_{1}}\right)-G_{\varepsilon_{2}}^{l}\left(x, t, \mathbf{u}_{\varepsilon_{2}}\right) \\
\quad \text { if } \min \left(\varphi_{\varepsilon_{1}}^{k}(t), \varphi_{\varepsilon_{2}}^{k}(t)\right)<x<\max \left(\varphi_{\varepsilon_{1}}^{k}(t)+\varepsilon_{1}, \varphi_{\varepsilon_{2}}^{k}(t)+\varepsilon_{2}\right) \quad \text { for } k \in\{1, \ldots, n\},
\end{array}\right.
\end{aligned}
$$

then it follows from Cauchy?s inequality that, for any $\sigma>0$,

$$
\begin{aligned}
& I_{3,1}^{l} \leq \sigma \iint_{Q_{\tau}}\left|\mathbf{w}_{x}\right|^{2} \mathrm{~d} x \mathrm{~d} t+C(\sigma) I_{3,4} \\
& I_{3,2}^{l} \leq \sigma \iint_{Q_{\tau}}\left|\mathbf{w}_{x}\right|^{2} \mathrm{~d} x \mathrm{~d} t+C(\sigma) \iint_{Q_{\tau}}|\mathbf{w}|^{2} \mathrm{~d} x \mathrm{~d} t+C I_{3,4}+C I_{3,5}
\end{aligned}
$$

and

$$
\begin{aligned}
I_{3,3}^{l} \leq & \sum_{k=1}^{n+1} \iint_{Q_{\tau}}\left|g_{k}^{l}\left(\mathbf{u}_{\varepsilon_{1}}\right)-g_{k}^{l}\left(\mathbf{u}_{\varepsilon_{2}}\right)\right|\left|w^{l}\right| \mathrm{d} x \mathrm{~d} t \\
& +\sum_{k=1}^{n} \int_{0}^{\tau} \int_{\min \left\{\varphi_{\varepsilon_{1}}^{k}, \varphi_{\varepsilon_{2}}^{k}\right\}}^{\max \left\{\varphi_{\varepsilon_{1}}^{k}+\varepsilon_{1}, \varphi_{\varepsilon_{2}}^{k}+\varepsilon_{2}\right\}}\left|G_{\varepsilon_{1}}^{l}\left(x, t, \mathbf{u}_{\varepsilon_{1}}\right)-G_{\varepsilon_{2}}^{l}\left(x, t, \mathbf{u}_{\varepsilon_{2}}\right)\right|\left|w^{l}\right| \mathrm{d} x \mathrm{~d} t \\
\leq & C \iint_{Q_{\tau}}|\mathbf{w}|^{2} \mathrm{~d} x \mathrm{~d} t+C \sum_{k=1}^{n}\left\|\left|\varphi_{\varepsilon_{1}}^{k}-\varphi_{\varepsilon_{2}}^{k}\right|+\varepsilon_{1}+\varepsilon_{2}\right\|_{L^{2}(0, \tau)},
\end{aligned}
$$


where

$$
\begin{aligned}
& I_{3,4}:=\sum_{l=1}^{n} \iint_{Q_{\tau}}\left[1+\sum_{j=1}^{2}\left(u_{\varepsilon_{j} x}^{l}\right)^{2}\right]\left[s_{\varepsilon_{1}}\left(x-\varphi_{\varepsilon_{1}}^{l}(t)\right)-s_{\varepsilon_{2}}\left(x-\varphi_{\varepsilon_{2}}^{l}(t)\right)\right]^{2} \mathrm{~d} x \mathrm{~d} t, \\
& I_{3,5}:=\sum_{l=1}^{N} \iint_{Q_{\tau}}\left[1+\sum_{j=1}^{2}\left(u_{\varepsilon_{j} x}^{l}\right)^{2}\right]\left(w^{l}\right)^{2} \mathrm{~d} x \mathrm{~d} t .
\end{aligned}
$$

According to the definition of function $s_{\varepsilon}(\theta)$, we see that $s_{\varepsilon_{1}}\left(x-\varphi_{\varepsilon_{1}}^{l}(t)\right)-s_{\varepsilon_{2}}\left(x-\varphi_{\varepsilon_{2}}^{l}(t)\right)=0$ if $x<\min \left\{\varphi_{\varepsilon_{1}}^{l}(t), \varphi_{\varepsilon_{2}}^{l}(t)\right\}$ or $x>\max \left\{\varphi_{\varepsilon_{1}}^{l}(t)+\varepsilon_{1}, \varphi_{\varepsilon_{2}}^{l}(t)+\varepsilon_{2}\right\}$. Thus by (3.21) we have

$$
\begin{aligned}
I_{3,4} \leq & \sum_{l=1}^{n}\left\{\int_{0}^{\tau}\left(1+\sum_{l, j=1}^{2}\left\|u_{\varepsilon_{j} x}^{l}\right\|_{L^{\infty}(0, d)}^{2}\right)^{2} \mathrm{~d} t\right\}^{1 / 2} \\
& \times\left\{\int_{0}^{\tau}\left[\int_{0}^{d}\left(s_{\varepsilon_{1}}\left(x-\varphi_{\varepsilon_{1}}^{l}(t)\right)-s_{\varepsilon_{2}}\left(x-\varphi_{\varepsilon_{2}}^{l}(t)\right)\right)^{2} \mathrm{~d} x\right]^{2} \mathrm{~d} t\right\}^{1 / 2} \\
\leq & C\left(1 / \delta,\left\|\boldsymbol{\varphi}_{t}\right\|_{\mathbf{L}^{2}(0, T)}\right) \sum_{l=1}^{n}\left\{\int_{0}^{\tau}\left[\int_{\min \left\{\varphi_{\varepsilon_{1}}^{l}, \varphi_{\varepsilon_{2}}^{l}\right\}}^{\max \left\{\varphi_{\varepsilon_{1}}^{l}+\varepsilon_{1}, \varphi_{\varepsilon_{2}}^{l}+\varepsilon_{2}\right\}} 1 \mathrm{~d} x\right]^{2} \mathrm{~d} t\right\}^{1 / 2} \\
\leq & C\left(1 / \delta,\left\|\boldsymbol{\varphi}_{t}\right\|_{\mathbf{L}^{2}(0, T)}\right) \sum_{l=1}^{n}\left\|\left|\varphi_{\varepsilon_{1}}^{l}-\varphi_{\varepsilon_{2}}^{l}\right|+\varepsilon_{1}+\varepsilon_{2}\right\|_{L^{2}(0, \tau)}
\end{aligned}
$$

and

$$
I_{3,5} \leq \int_{0}^{\tau}\left[\left(1+\sum_{j=1}^{2}\left\|\mathbf{u}_{\varepsilon_{j} x}\right\|_{\mathbf{L}^{\infty}(0, d)}^{2}\right) \int_{0}^{d}|\mathbf{w}|^{2} \mathrm{~d} x\right] \mathrm{d} t .
$$

Summing equality (3.30) with respect to $l$ from $l=1$ to $l=N$, using (3.31)-(3.35) and Minkowski?s inequality, and choosing $\sigma=v / 8$, we then conclude that

$$
\begin{aligned}
& \int_{0}^{d}|\mathbf{w}|^{2}(x, \tau) \mathrm{d} x+\int_{0}^{\tau} \int_{0}^{d}\left|\mathbf{w}_{x}\right|^{2} \mathrm{~d} x \mathrm{~d} t \\
& \quad \leq C\left(1 / \delta,\left\|\boldsymbol{\varphi}_{t}\right\|_{\mathbf{L}^{2}(0, T)}\right)\left\{\int_{0}^{\tau}\left[\left(1+\sum_{j=1}^{2}\left\|\mathbf{u}_{\varepsilon_{j} x}\right\|_{\mathbf{L}^{\infty}(0, d)}^{2}\right) \int_{0}^{d}|\mathbf{w}|^{2} \mathrm{~d} x\right] \mathrm{d} t+I_{3,0}\right\} .
\end{aligned}
$$

This, together with Gronwall?s inequality, yields

$$
\begin{aligned}
\int_{0}^{\tau} & {\left[\left(1+\sum_{j=1}^{2}\left\|\mathbf{u}_{\varepsilon_{j} x}\right\|_{\mathbf{L}^{\infty}(0, d)}^{2}\right) \int_{0}^{d}|\mathbf{w}|^{2} \mathrm{~d} x\right] \mathrm{d} t } \\
\leq & C\left(1 / \delta,\left\|\boldsymbol{\varphi}_{t}\right\|_{\mathbf{L}^{2}(0, T)}\right) I_{3,0} \exp \left\{C \int_{0}^{T}\left(1+\sum_{j=1}^{2}\left\|\mathbf{u}_{\varepsilon_{j} x}\right\|_{\mathbf{L}^{\infty}(0, d)}^{2}\right) \mathrm{d} t\right\} \\
& \times \int_{0}^{T}\left(1+\sum_{j=1}^{2}\left\|\mathbf{u}_{\varepsilon_{j} x}\right\|_{\mathbf{L}^{\infty}(0, d)}^{2}\right) \mathrm{d} t .
\end{aligned}
$$

Combining the two inequalities above and (3.21) leads us to estimate (3.28). 


\subsection{The solutions of the diffraction problem}

Proof of Theorem 2.1 We divide the proof into three steps.

Step 1. We prove the global existence of the solutions. Let us discuss the behavior of the solution $\mathbf{u}_{\varepsilon}$ associated with $\boldsymbol{\varphi}_{\varepsilon}(t)$ by Theorem 2.1 as $\varepsilon \rightarrow 0$.

We first see from (3.16) that for any $\tau \in[0, T]$ and any vector function $\eta \in \mathcal{W}_{2}^{1,1}\left(\mathcal{Q}_{T}\right)$,

$$
\begin{aligned}
& \left.\int_{0}^{d} u_{\varepsilon}^{l} \eta^{l} \mathrm{~d} x\right|_{0} ^{\tau}+\iint_{Q_{\tau}}\left\{-u_{\varepsilon}^{l} \eta_{t}^{l}+A_{\varepsilon}^{l}(x, t) u_{\varepsilon x}^{l} \eta_{x}^{l}-\left[B_{\varepsilon}^{l}\left(x, t, u_{\varepsilon}^{l}\right) u_{\varepsilon x}^{l}+G_{\varepsilon}^{l}\left(x, t, \mathbf{u}_{\varepsilon}\right)\right] \eta^{l}\right\} \mathrm{d} x \mathrm{~d} t \\
& \quad=0, \quad l=1, \ldots, N .
\end{aligned}
$$

Furthermore, according to estimates (3.17), (3.20), (3.28) and the Arzela-Ascoli theorem, we conclude that there exists a subsequence (we retain the same notation for it) $\left\{\mathbf{u}_{\varepsilon}\right\}$ such that

$$
\begin{aligned}
& \mathbf{u}_{\varepsilon} \rightarrow \mathbf{u} \quad \text { in } \mathcal{C}\left(\bar{Q}_{T}\right), \quad \mathbf{u}_{\varepsilon t} \rightarrow \mathbf{u}_{t} \quad \text { weakly in } \mathcal{L}^{2}\left(Q_{T}\right), \\
& \mathbf{u}_{\varepsilon} \rightarrow \mathbf{u} \quad \text { in } \mathcal{V}_{2}^{1,0}\left(\mathcal{Q}_{T}\right) .
\end{aligned}
$$

Thus $\mathbf{u}$ is in $\mathcal{C}^{\alpha_{1}, \alpha_{1} / 2}\left(\overline{\mathcal{Q}}_{T}\right)$, $\mathbf{u}$ satisfies the parabolic condition (1.2), and estimates (2.6) and (2.7) hold.

We next show that for each $l=1, \ldots, N$, the sequences $\left\{A_{\varepsilon}^{l}(x, t) u_{\varepsilon x}^{l}\right\},\left\{B_{\varepsilon}^{l}\left(x, t, u_{\varepsilon}^{l}\right) u_{\varepsilon x}^{l}\right\}$, $\left\{G_{\varepsilon}^{l}\left(x, t, \mathbf{u}_{\varepsilon}\right)\right\}$ converge in $L^{2}\left(Q_{T}\right)$ to $A^{l}(x, t) u_{x}^{l}, B^{l}\left(x, t, u^{l}\right) u_{x}^{l}$ and $G^{l}(x, t, \mathbf{u})$, respectively. Since

$$
\begin{aligned}
& \operatorname{mes}\left\{(x, t): \min \left(\varphi_{\varepsilon}^{i}(t), \varphi^{i}(t)\right) \leq x \leq \max \left(\varphi_{\varepsilon}^{i}(t)+\varepsilon, \varphi^{i}(t)+\varepsilon\right), 0 \leq t \leq T\right\} \\
& \quad \rightarrow 0 \quad \text { as } \varepsilon \rightarrow 0, i=1, \ldots, n,
\end{aligned}
$$

and

$$
\left(u_{\varepsilon x}^{l}\right)^{2}=\left(u_{\varepsilon x}^{l}-u_{x}^{l}+u_{x}^{l}\right)^{2} \leq 2\left(u_{l x}^{\varepsilon}-u_{x}^{l}\right)^{2}+2\left(u_{x}^{l}\right)^{2}
$$

then it follows from (2.2), (3.3) and Lebesgue dominated convergence theorem that

$$
\begin{aligned}
& \iint_{Q_{T}}\left[B_{\varepsilon}^{l}\left(x, t, u_{\varepsilon}^{l}\right) u_{\varepsilon x}^{l}-B^{l}\left(x, t, u^{l}\right) u_{x}^{l}\right]^{2} \mathrm{~d} x \mathrm{~d} t \\
& =\int_{0}^{T}\left\{\int_{0}^{\min \left(\varphi_{\varepsilon}^{l}, \varphi^{l}\right)}\left[\hat{b}\left(u_{\varepsilon}^{l}\right) u_{\varepsilon x}^{l}-\hat{b}\left(u^{l}\right) u_{x}^{l}\right]^{2}\right. \\
& \quad+\int_{\max \left(\varphi_{\varepsilon}^{l}+\varepsilon, \varphi^{l}+\varepsilon\right)^{d}}^{d}\left[\tilde{b}\left(u_{\varepsilon}^{l}\right) u_{\varepsilon x}^{l}-\tilde{b}\left(u^{l}\right) u_{x}^{l}\right]^{2} \\
& \left.\quad+\int_{\min \left(\varphi_{\varepsilon}^{l}, \varphi^{l}\right)}^{\max \left(\varphi_{\varepsilon}^{l}+\varepsilon, \varphi^{l}+\varepsilon\right)}\left[B_{\varepsilon}^{l}\left(x, t, u_{\varepsilon}^{l}\right) u_{\varepsilon x}^{l}-B^{l}\left(x, t, u^{l}\right) u_{x}^{l}\right]^{2} \mathrm{~d} x\right\} \mathrm{d} t \\
& \leq C \iint_{Q_{T}}\left(u_{\varepsilon}^{l}-u^{l}\right)^{2}\left(1+\left|u_{x}^{l}\right|\right)^{2} \mathrm{~d} x \mathrm{~d} t+C \iint_{Q_{T}}\left(u_{\varepsilon x}^{l}-u_{x}^{l}\right)^{2} \mathrm{~d} x \mathrm{~d} t \\
& \quad+C \int_{0}^{T} \int_{\min \left(\varphi_{\varepsilon}^{l}, \varphi^{l}\right)}^{\max \left(\varphi_{\varepsilon}^{l}+\varepsilon, \varphi^{l}+\varepsilon\right)}\left[\left(u_{\varepsilon x}^{l}-u_{x}^{l}\right)^{2}+\left(u_{x}^{l}\right)^{2}+1\right] \mathrm{d} x \mathrm{~d} t \\
& \quad \rightarrow 0 \quad \text { as } \varepsilon \rightarrow 0, l \in\{1, \ldots, n\},
\end{aligned}
$$


and

$$
\begin{aligned}
& \iint_{Q_{T}}\left[B_{\varepsilon}^{l}\left(x, t, u_{\varepsilon}^{l}\right) u_{\varepsilon x}^{l}-B^{l}\left(x, t, u^{l}\right) u_{x}^{l}\right]^{2} \mathrm{~d} x \mathrm{~d} t \\
& \quad=\iint_{Q_{T}}\left[b^{l}\left(u_{\varepsilon}^{l}\right) u_{\varepsilon x}^{l}-b^{l}\left(u^{l}\right) u_{x}^{l}\right]^{2} \mathrm{~d} x \mathrm{~d} t \\
& \quad \leq C \iint_{Q_{T}}\left(u_{\varepsilon}^{l}-u^{l}\right)^{2}\left(1+\left|u_{x}^{l}\right|\right)^{2} \mathrm{~d} x \mathrm{~d} t+C \iint_{Q_{T}}\left(u_{\varepsilon x}^{l}-u_{x}^{l}\right)^{2} \mathrm{~d} x \mathrm{~d} t \\
& \quad \rightarrow 0 \quad \text { as } \varepsilon \rightarrow 0, l \in\{n+1, \ldots, N\},
\end{aligned}
$$

and from (3.10) that

$$
\begin{aligned}
& \iint_{Q_{T}}\left[G_{\varepsilon}^{l}\left(x, t, \mathbf{u}_{\varepsilon}\right)-G^{l}(x, t, \mathbf{u})\right]^{2} \mathrm{~d} x \mathrm{~d} t \\
& \quad \leq \sum_{k=1}^{n+1} \iint_{Q_{T}}\left[g_{k}^{l}\left(\mathbf{u}_{\varepsilon}\right)-g_{k}^{l}(\mathbf{u})\right]^{2} \mathrm{~d} x \mathrm{~d} t \\
& \quad+\sum_{k=1}^{n} \int_{0}^{\tau} \int_{\min \left\{\varphi_{\varepsilon}^{k}, \varphi^{k}\right\}}^{\max \left\{\varphi^{k}+\varepsilon, \varphi^{k}+\varepsilon\right\}}\left[G_{\varepsilon}^{l}\left(x, t, \mathbf{u}_{\varepsilon}\right)-G^{l}(x, t, \mathbf{u})\right]^{2} \mathrm{~d} x \mathrm{~d} t \\
& \leq C \iint_{Q_{T}}\left|\mathbf{u}_{\varepsilon}-\mathbf{u}\right|^{2} \mathrm{~d} x \mathrm{~d} t+C \sum_{k=1}^{n} \int_{0}^{\tau} \int_{\min \left\{\varphi_{\varepsilon}^{k}, \varphi^{k}\right\}}^{\max \left\{\varphi_{\varepsilon}^{k}+\varepsilon, \varphi^{k}+\varepsilon\right\}} 1 \mathrm{~d} x \mathrm{~d} t \\
& \quad \rightarrow 0, \quad l \in\{1, \ldots, N\} .
\end{aligned}
$$

The similar argument shows that $\left\|A_{\varepsilon}^{l}(x, t) u_{\varepsilon x}^{l}-A^{l}(x, t) u_{x}^{l}\right\|_{L^{2}\left(Q_{T}\right)} \rightarrow 0$ for each $l=1, \ldots, N$.

Based on the above arguments for sequences $\left\{A_{\varepsilon}^{l}(x, t) u_{\varepsilon x}^{l}\right\},\left\{B_{\varepsilon}^{l}\left(x, t, u_{\varepsilon}^{l}\right) u_{\varepsilon x}^{l}\right\},\left\{G_{\varepsilon}^{l}\left(x, t, \mathbf{u}_{\varepsilon}\right)\right\}$ and $\left\{u_{\varepsilon t}^{l}\right\}$, by letting $\varepsilon \rightarrow 0$, we conclude from (3.36) that (2.4) holds.

We also see from (3.20) that there exists a subsequence $\left\{\mathbf{u}_{\varepsilon^{\prime}}\right\}$ (denoted by $\left\{\mathbf{u}_{\varepsilon}\right\}$ still) such that for each $l=1, \ldots, N,\left\{\left(A_{\varepsilon}(x, t) u_{\varepsilon x}^{l}\right)_{x}\right\}$ converge weakly in $L^{2}\left(Q_{T}\right)$ to $\varpi^{l}(x, t)$. Recalling that $A_{\varepsilon}(x, t) u_{\varepsilon x}^{l} \rightarrow A(x, t) u_{x}^{l}$ in $L^{2}\left(Q_{T}\right)$, we deduce $\varpi_{l}=\left(A(x, t) u_{x}^{l}\right)_{x}$. These, together with (3.20), imply that $u_{x x}^{l} \in L^{2}\left(Q_{T}^{l-}\right) \cap L^{2}\left(Q_{T}^{l+}\right)$ for each $l=1, \ldots, n, u_{x x}^{l} \in L^{2}\left(Q_{T}\right)$ for each $l=$ $n+1, \ldots, N, A^{l}(x, t) u_{x}^{l} \in V_{2}\left(Q_{T}\right)$ for each $l=1, \ldots, N$, and (2.8) holds. Thus (2.4) implies that $\mathbf{u}$ satisfies the equations in (1.1a) and (1.1b) for almost all $(x, t) \in Q_{T}$ and the inner boundary condition (1.3) for almost all $t \in[0, T]$ (see [17, Chapter 3, Section 13]). As we have done in the derivation of (3.21), estimate (2.8) yields (2.9).

For fixed $l \in\{1, \ldots, N\}$ and $k \in\{1, \ldots, n+1\}, u^{l}$ satisfies the linear equation

$$
u_{t}^{l}-\underline{a}^{l} u_{x x}^{l}=\underline{b}^{l}(x, t) u_{x}^{l}+\underline{g}^{l}(x, t) \quad\left((x, t) \in Q_{k, T}\right),
$$

where

$$
\underline{a}^{l}:=\left\{\begin{array}{ll}
\hat{a}^{l} & k \leq l, l=1, \ldots, n, \\
\tilde{a}^{l} & k>l, l=1, \ldots, n, \\
a^{l} & l=n+1, \ldots, N,
\end{array} \quad \underline{b}^{l}(x, t):= \begin{cases}\hat{b}^{l}\left(u^{l}(x, t)\right) & k \leq l, l=1, \ldots, n, \\
\tilde{b}^{l}\left(u^{l}(x, t)\right) & k>l, l=1, \ldots, n, \\
b^{l}\left(u^{l}(x, t)\right) & l=n+1, \ldots, N,\end{cases}\right.
$$

and

$$
\underline{g}^{l}(x, t):=g_{k}^{l}(\mathbf{u}(x, t))
$$


Then for any subdomains $Q^{\prime}$ and $Q^{\prime \prime}$ satisfying $\bar{Q} \subset Q^{\prime \prime}$ and $\bar{Q}^{\prime \prime} \subset Q_{k, T}$, we have $\underline{b}^{l}(x, t)$, $\underline{g}(x, t) \in C^{\alpha_{1}, \alpha_{1} / 2}\left(\bar{Q}^{\prime \prime}\right)$. The parabolic regularity theory shows that $u^{l} \in C^{2+\alpha_{1}, 1+\alpha_{1} / 2}\left(\bar{Q}^{\prime}\right)$. Hence $\mathbf{u}$ satisfies pointwise the equations in (1.1a) and (1.1b) for $(x, t) \in Q_{k, T}$. Consequently, $\mathbf{u}$ is a solution in $\mathscr{S}$ of problem (1.1a)-(1.3) and estimates (2.6)-(2.9) hold.

Step 2. In what follows, we will show that the solution in $\mathscr{S}$ for problem (1.1a)-(1.3) is unique and estimates (2.10) and (2.11) hold.

Let $\mathbf{u}_{1}, \mathbf{u}_{2}$ be the solutions in $\mathscr{S}$ corresponding to $\varphi_{1}$ and $\varphi_{2}$, respectively. Set $\mathbf{w}=\mathbf{u}_{1}-\mathbf{u}_{2}$. Then $\mathbf{w} \in \mathcal{W}_{2}^{1,1}\left(Q_{T}\right)$. We choose $\boldsymbol{\eta}=\mathbf{w}$ in (2.4) to find

$$
\begin{aligned}
& \iint_{Q_{\tau}} u_{i t}^{l} w^{l} \mathrm{~d} x \mathrm{~d} t+\int_{0}^{\tau}\left\{\int_{0}^{\varphi_{i}^{l}(t)}\left[\hat{a}^{l} u_{i x}^{l} w_{x}^{l}-\hat{b}^{l}\left(u_{i}^{l}\right) u_{i x}^{l} w^{l}\right] \mathrm{d} x\right. \\
& \left.\quad+\int_{\varphi_{i}^{l}(t)}^{d}\left[\tilde{a}^{l} u_{i x}^{l} w_{x}^{l}-\tilde{b}^{l}\left(u_{i}^{l}\right) u_{i x}^{l} w^{l}\right] \mathrm{d} x\right\}-\sum_{k=1}^{n+1} \int_{0}^{\tau} \int_{\varphi_{i}^{k-1}(t)}^{\varphi_{i}^{k}(t)} g_{k}^{l}\left(\mathbf{u}_{i}\right) w^{l} \mathrm{~d} x \mathrm{~d} t \\
& =0, \quad i=1,2, l=1, \ldots, n,
\end{aligned}
$$

and

$$
\begin{aligned}
& \iint_{Q_{\tau}}\left[u_{i t}^{l} w^{l}+a^{l} u_{i x}^{l} w_{x}^{l}-b^{l}\left(u_{i}^{l}\right) u_{i x}^{l} w^{l}\right] \mathrm{d} x \mathrm{~d} t-\sum_{k=1}^{n+1} \int_{0}^{\tau} \int_{\varphi_{i}^{k-1}(t)}^{\varphi_{i}^{k}(t)} g_{k}^{l}\left(\mathbf{u}_{i}\right) w^{l} \mathrm{~d} x \mathrm{~d} t \\
& \quad=0, \quad i=1,2, l=n+1, \ldots, N .
\end{aligned}
$$

For each $l=1, \ldots, N$, by a subtraction of the above equations for $i=1,2$, we conclude that

$$
\begin{aligned}
& \frac{1}{2} \int_{0}^{d}\left(w^{l}(x, \tau)\right)^{2} \mathrm{~d} x+\int_{0}^{\tau}\left\{\int_{0}^{\varphi_{1}^{l}}\left[\hat{a}^{l}\left(w_{x}^{l}\right)^{2}-\left(\hat{b}^{l}\left(u_{1}^{l}\right) u_{1 x}^{l}-\hat{b}^{l}\left(u_{2}^{l}\right) u_{2 x}^{l}\right) w^{l}\right] \mathrm{d} x\right. \\
& \quad+\int_{\varphi_{1}^{l}}^{d}\left[\tilde{a}^{l}\left(w_{x}^{l}\right)^{2}-\left(\tilde{b}^{l}\left(u_{1}^{l}\right) u_{1 x}^{l}-\tilde{b}^{l}\left(u_{2}^{l}\right) u_{2 x}^{l}\right) w^{l}\right] \mathrm{d} x \\
& \left.\quad+\int_{\varphi_{1}^{l}}^{\varphi_{2}^{l}}\left(\hat{b}^{l}\left(u_{2}^{l}\right)-\tilde{b}^{l}\left(u_{2}^{l}\right)\right) u_{2 x}^{l} w^{l} \mathrm{~d} x\right\} \mathrm{d} t \\
& =\sum_{k=1}^{n+1} \int_{0}^{\tau}\left\{\int_{\varphi_{2}^{k-1}(t)}^{\varphi_{2}^{k}(t)}\left[g_{k}^{l}\left(\mathbf{u}_{1}\right)-g_{k}^{l}\left(\mathbf{u}_{2}\right)\right] w^{l} \mathrm{~d} x\right. \\
& \left.\quad+\left(\int_{\varphi_{1}^{k-1}(t)}^{\varphi_{2}^{k-1}(t)}+\int_{\varphi_{2}^{k}(t)}^{\varphi_{1}^{k}(t)}\right) g_{k}^{l}\left(\mathbf{u}_{1}\right) w^{l} \mathrm{~d} x\right\} \mathrm{d} t, \quad l \in\{1, \ldots, n\},
\end{aligned}
$$

and

$$
\begin{aligned}
& \frac{1}{2} \int_{0}^{d}\left(w^{l}(x, \tau)\right)^{2} \mathrm{~d} x+\iint_{Q_{\tau}}\left[a^{l}\left(w_{x}^{l}\right)^{2}+\left(b^{l}\left(u_{1}^{l}\right) u_{1 x}^{l}-b^{l}\left(u_{2}^{l}\right) u_{2 x}^{l}\right) w^{l}\right] \mathrm{d} x \\
& =\sum_{k=1}^{n+1} \int_{0}^{\tau}\left\{\int_{\varphi_{2}^{k-1}(t)}^{\varphi_{2}^{k}(t)}\left[g_{k}^{l}\left(\mathbf{u}_{1}\right)-g_{k}^{l}\left(\mathbf{u}_{2}\right)\right] w^{l} \mathrm{~d} x\right. \\
& \left.\quad+\left(\int_{\varphi_{1}^{k-1}(t)}^{\varphi_{2}^{k-1}(t)}+\int_{\varphi_{2}^{k}(t)}^{\varphi_{1}^{k}(t)}\right) g_{k}^{l}\left(\mathbf{u}_{1}\right) w^{l} \mathrm{~d} x\right\} \mathrm{d} t, \quad l \in\{n+1, \ldots, N\} .
\end{aligned}
$$


Then

$$
\begin{aligned}
& \int_{0}^{d}\left(w^{l}(x, \tau)\right)^{2} \mathrm{~d} x+\iint_{Q_{\tau}}\left(w_{x}^{l}\right)^{2} \mathrm{~d} x \mathrm{~d} t \\
& \leq C \iint_{Q_{\tau}}\left[\left|w_{x}^{l} w^{l}\right|+\left|u_{1 x}^{l}\right|\left(w^{l}\right)^{2}\right] \mathrm{d} x \mathrm{~d} t \\
& \quad+C\left|\int_{0}^{\tau} \int_{\varphi_{1}^{l}(t)}^{\varphi_{2}^{l}(t)}\right| u_{2 x}^{l}|| w^{l}|\mathrm{~d} x \mathrm{~d} t|+\sum_{k=1}^{n+1} \iint_{Q_{\tau}}\left|g_{k}^{l}\left(\mathbf{u}_{1}\right)-g_{k}^{l}\left(\mathbf{u}_{2}\right)\right|\left|w^{l}\right| \mathrm{d} x \mathrm{~d} t \\
& \quad+C \sum_{k=1}^{n} \int_{0}^{\tau} \int_{\varphi_{1}^{k}(t)}^{\varphi_{2}^{k}(t)}\left|w^{l}\right| \mathrm{d} x \mathrm{~d} t \\
& \leq \epsilon \int_{Q_{\tau}}\left|w_{x}^{l}\right|^{2} \mathrm{~d} x \mathrm{~d} t+C(\epsilon) \iint_{Q_{\tau}}\left[1+\sum_{j=1}^{2}\left(u_{j x}^{l}\right)^{2}\right]|\mathbf{w}|^{2} \mathrm{~d} x \mathrm{~d} t \\
& \quad+C \sum_{k=1}^{n} \int_{0}^{\tau}\left|\varphi_{1}^{k}(t)-\varphi_{2}^{k}(t)\right| \mathrm{d} t, \quad l=1, \ldots, N .
\end{aligned}
$$

Setting $\epsilon=1 / 2$ and summing the above inequalities with respect to $l$ from $l=1$ to $l=N$, we have

$$
\begin{aligned}
& \int_{0}^{d}|\mathbf{w}(x, \tau)|^{2} \mathrm{~d} x+\iint_{Q_{\tau}}\left|\mathbf{w}_{x}\right|^{2} \mathrm{~d} x \mathrm{~d} t \\
& \quad \leq C \iint_{Q_{\tau}}\left[1+\sum_{j=1}^{2}\left|\mathbf{u}_{j x}\right|^{2}\right]|\mathbf{w}|^{2} \mathrm{~d} x \mathrm{~d} t+C\left\|\boldsymbol{\varphi}_{1}-\boldsymbol{\varphi}_{2}\right\|_{L^{2}(0, T)} \\
& \quad \leq C \int_{0}^{\tau}\left[\left(1+\sum_{j=1}^{2}\left\|\mathbf{u}_{\varepsilon_{j} x}\right\|_{L^{\infty}(0, d)}^{2}\right) \int_{0}^{d}|\mathbf{w}|^{2} \mathrm{~d} x\right] \mathrm{d} t+C\left\|\boldsymbol{\varphi}_{1}-\boldsymbol{\varphi}_{2}\right\|_{L^{2}(0, T)} .
\end{aligned}
$$

Again by Gronwall?s inequality we deduce 2.10), which, together with [17, Chapter 2, formula (3.8)], gives (2.11). Therefore the solution in $\mathscr{S}$ for problem (1.1a)-(1.3) associated with $\varphi(t)$ is unique.

Step 3. For $\boldsymbol{\varphi} \in \mathbf{C}^{2}((0, T])$, we discuss the regularity of $\mathbf{u}$.

For any fixed $l \in\{1, \ldots, n\}$, let

$$
\begin{aligned}
& y=x-\varphi^{l}(t), \quad t^{\prime}=t \quad(\text { denoted by } t \text { still }), \\
& v(y, t)=u^{l}\left(y+\varphi^{l}(t), t\right)
\end{aligned}
$$

Then $v$ satisfies

$$
\begin{aligned}
& v_{t}=\mathcal{H}(y, t, v) \\
& \quad:=\left\{\begin{array}{lr}
\hat{a}^{l} v_{y y}+v_{y} \varphi_{t}^{l}+\hat{b}^{l}(v) v_{y}+g_{l}^{l}\left(\mathbf{u}\left(y+\varphi^{l}(t), t\right)\right) & ((x, t) \in(-\delta, 0) \times(0, T]), \\
\tilde{a}^{l} v_{y y}+v_{y} \varphi_{t}^{l}+\tilde{b}^{l}(v) v_{y}+g_{l+1}^{l}\left(\mathbf{u}\left(y+\varphi^{l}(t), t\right)\right) & ((x, t) \in(0, \delta) \times(0, T]),
\end{array}\right. \\
& v^{-}(0, t)=v^{+}(0, t), \quad \hat{a}^{l} v_{y}^{-}(0, t)=\tilde{a}^{l} v_{y}^{+}(0, t) \quad(t \in[0, T]) .
\end{aligned}
$$


We will use the result of [18] to obtain the regularity of $v$. To do this, we need the estimate of

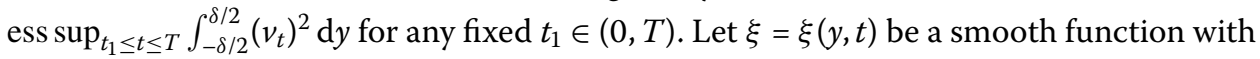
values between 0 and 1 such that $\xi=0$ for $|y| \geq \delta$ or $t \leq t_{1} / 2, \xi=1$ for $(y, t) \in[-\delta / 2, \delta / 2] \times$ $\left[t_{1}, T\right]$ and $\left|\xi_{y}\right|+\left|\xi_{t}\right| \leq C\left(1 / \delta, 1 / t_{1}\right)$, and let

$$
v_{(t)}=[v(x, t+\Delta t)-v(x, t)] / \Delta t .
$$

For any small enough $\Delta t$, consider the equality $\int_{t_{1} / 2}^{\tau} \int_{-\delta}^{\delta}\left[v_{t}-\mathcal{H}(y, t, v)\right]_{(t)} v_{(t)}(\xi)^{2} \mathrm{~d} x \mathrm{~d} t$. By employing the formula of integration by parts and [17, Chapter II, formula (4.7)], we get

$$
\begin{aligned}
0= & \frac{1}{2} \int_{-\delta}^{\delta}\left(v_{(t)}(y, \tau) \xi(y, \tau)\right)^{2} \mathrm{~d} y-\int_{t_{1} / 2}^{\tau} \int_{-\delta}^{\delta}\left(v_{(t)}\right)^{2} \xi \xi_{t} \mathrm{~d} y \mathrm{~d} t \\
& +\int_{t_{1} / 2}^{\tau}\left\{\int _ { - \delta } ^ { 0 } \left[\left(\hat{a}^{l}\left(v_{y(t)} \xi\right)^{2}+2 \hat{a}^{l} v_{y(t)} v_{(t)} \xi \xi_{y}\right)+\left(\left(\varphi_{t}^{l}\right)_{(t)} v_{y}+\varphi_{t}(t+\Delta t) v_{y(t)}\right) v_{(t)}(\xi)^{2}\right.\right. \\
& \left.+\left(\left(\hat{b}^{l}(v)\right)_{(t)} v_{y}+\hat{b}^{l}(v(y, t+\Delta t)) v_{y(t)}\right) v_{(t)}(\xi)^{2}+\left(g_{l}^{l}\left(\mathbf{u}\left(y+\varphi^{l}(t), t\right)\right)\right)_{(t)} v_{(t)}(\xi)^{2}\right] \mathrm{d} y \\
& +\int_{0}^{\delta}\left[\left(\tilde{a}^{l}\left(v_{y(t)} \xi\right)^{2}+2 \tilde{a}^{l} v_{y(t)} v_{(t)} \xi \xi_{y}\right)+\left(\left(\varphi_{t}^{l}\right)_{(t)} v_{y}+\varphi_{t}(t+\Delta t) v_{y(t)}\right) v_{(t)}(\xi)^{2}\right. \\
& +\left(\left(\tilde{b}^{l}(v)\right)_{(t)} v_{y}+\tilde{b}^{l}(v(y, t+\Delta t)) v_{y(t)}\right) v_{(t)}(\xi)^{2} \\
& \left.\left.+\left(g_{l+1}^{l}\left(\mathbf{u}\left(y+\varphi^{l}(t), t\right)\right)\right)_{(t)} v_{(t)}(\xi)^{2}\right] \mathrm{~d} y\right\} \mathrm{d} t,
\end{aligned}
$$

where $v_{y(t)}=\left(v_{y}\right)_{(t)}$. Some tedious computation and Cauchy?s inequality yield

$$
\begin{aligned}
& \frac{1}{2} \int_{-\delta}^{\delta}\left(v_{(t)}(y, \tau) \xi(y, \tau)\right)^{2} \mathrm{~d} y+v_{0} \int_{t_{1} / 2}^{\tau} \int_{-\delta}^{\delta}\left(v_{y(t)} \xi\right)^{2} \mathrm{~d} y \mathrm{~d} t \\
& \leq \epsilon \int_{t_{1} / 2}^{\tau} \int_{-\delta}^{\delta}\left(v_{y(t)} \xi\right)^{2} \mathrm{~d} y \mathrm{~d} t \\
& \quad+C\left(\epsilon, 1 / \delta, 1 / t_{1},\left\|\varphi^{l}\right\|_{C^{2}\left(\left[t_{1} / 2, T\right]\right)}\right) \int_{t_{1} / 2}^{\tau} \int_{-\delta}^{\delta}\left[1+\left(v_{y}\right)^{2}+\left(v_{(t)}\right)^{2}+\left(v_{(t)} \xi\right)^{2} v_{y}\right] \mathrm{d} y \mathrm{~d} t \\
& \quad+C\left(\left\|\varphi^{l}\right\|_{C^{1}\left(\left[t_{1} / 2, T\right]\right)}\right) \sum_{i=1}^{N} \iint_{Q_{T}}\left[\left(u_{x}^{i}(x, t)\right)^{2}+\left(u_{t}^{i}(x, t)\right)^{2}\right] \mathrm{d} x \mathrm{~d} t .
\end{aligned}
$$

We choose $\epsilon=\nu_{0} / 4$ and employ (2.8) to find

$$
\begin{aligned}
& \int_{-\delta}^{\delta}\left(v_{(t)}(y, \tau) \xi(y, \tau)\right)^{2} \mathrm{~d} y+\int_{t_{1} / 2}^{\tau} \int_{-\delta}^{\delta}\left(v_{y(t)} \xi\right)^{2} \mathrm{~d} y \mathrm{~d} t \\
& \quad \leq C\left(1 / \delta, 1 / t_{1},\left\|\varphi^{l}\right\|_{C^{2}([0, T])}\right)\left\{1+\int_{t_{1} / 2}^{\tau} \int_{-\delta}^{\delta}\left(v_{(t)} \xi\right)^{2} v_{y} \mathrm{~d} y \mathrm{~d} t\right\} \\
& \quad \leq C\left(1 / \delta, 1 / t_{1},\left\|\varphi^{l}\right\|_{C^{2}\left(\left[t_{1} / 2, T\right]\right)}\right)\left\{1+\int_{t_{1} / 2}^{\tau}\left\|v_{y}\right\|_{L^{\infty}(-\delta, \delta)} \int_{-\delta}^{\delta}\left(v_{(t)} \xi\right)^{2} \mathrm{~d} y \mathrm{~d} t\right\} .
\end{aligned}
$$

As we have done in the derivation of (3.20), by Gronwall?s inequality we get

$$
\int_{-\delta}^{\delta}\left(v_{(t)}(y, \tau) \xi(y, \tau)\right)^{2} \mathrm{~d} y+\int_{t_{1} / 2}^{\tau} \int_{-\delta}^{\delta}\left(v_{y(t)} \xi\right)^{2} \mathrm{~d} y \mathrm{~d} t \leq C\left(1 / \delta, 1 / t_{1},\left\|\varphi^{l}\right\|_{C^{2}([0, T])}\right) .
$$


Consequently,

$$
\int_{-\delta / 2}^{\delta / 2}\left(v_{(t)}\right)^{2} \mathrm{~d} y+\int_{t_{1}}^{\tau} \int_{-\delta / 2}^{\delta / 2}\left(v_{y(t)}\right)^{2} \mathrm{~d} y \mathrm{~d} t \leq C\left(1 / \delta, 1 / t_{1},\left\|\varphi^{l}\right\|_{C^{2}\left(\left[t_{1} / 2, T\right]\right)}\right) .
$$

By [17, Chapter II, Lemma 4.11], this inequality implies that

$$
\underset{t_{1} \leq t \leq T}{\operatorname{ess} \sup } \int_{-\delta / 2}^{\delta / 2}\left(v_{t}\right)^{2} \mathrm{~d} y+\int_{t_{1}}^{T} \int_{-\delta / 2}^{\delta / 2}\left(v_{y t}\right)^{2} \mathrm{~d} y \mathrm{~d} t \leq C\left(1 / \delta, 1 / t_{1},\left\|\varphi^{l}\right\|_{C^{2}\left(\left[t_{1} / 2, T\right]\right)}\right) .
$$

Hence $u_{x t}^{l} \in \mathrm{t}^{2}\left(Q^{l}\left(\delta, t_{1}\right)\right)$. Using (3.37), hypothesis (H)(iii) and [18, Theorem 1.1], we deduce that $v_{y}(y, t)$ is continuous with respect to $y$ in $[-\delta / 4,0]$ and in $[0, \delta / 4]$ for almost all $t \in\left[t_{1}, T\right]$, and $v_{t}$ is continuous in $[-\delta / 4, \delta / 4] \times\left[t_{1}, T\right]$. Since $u_{x}^{l}(x, t)=v_{y}\left(x-\varphi^{l}(t), t\right)$ and $u_{t}^{l}(x, t)=v_{t}\left(x-\varphi^{l}(t), t\right)-v_{y}\left(x-\varphi^{l}(t), t\right) \varphi_{t}^{l}(t)$, then for almost all $t \in\left[t_{1}, T\right], u_{x}^{l}(x, t)$ and $u_{t}^{l}(x, t)$ are continuous with respect to $x$ in $\left[\varphi^{l}(t)-\delta / 4, \varphi^{l}(t)\right]$ and in $\left[\varphi^{l}(t), \varphi^{l}(t)+\delta / 4\right]$.

The following corollary follows directly from Theorem 2.1.

Corollary 3.3 Assume that $\boldsymbol{\varphi}_{m}(t) \in \mathbf{C}^{2}([0, T]), m=1,2, \ldots$, satisfy $(2.5)$ and the sequence $\left\{\boldsymbol{\varphi}_{m}(t)\right\}$ converges in $\mathbf{W}_{2}^{1}(0, T)$ to $\boldsymbol{\varphi}(t)$. If $\mathbf{u}_{m}, \mathbf{u}$ are the solutions in $\mathscr{S}$ of (1.1a)-(1.3) corresponding to $\varphi_{m}$ and $\varphi$, respectively, then there exists a subsequence (we retain the same notation for it) $\left\{\mathbf{u}_{m}\right\}$ such that

$$
\mathbf{u}_{m} \rightarrow \mathbf{u} \quad \text { in } \mathcal{C}\left(\bar{Q}_{T}\right), \quad \mathbf{u}_{m t} \rightarrow \mathbf{u}_{t} \quad \text { weakly in } \mathcal{L}^{2}\left(Q_{T}\right), \quad \mathbf{u}_{m} \rightarrow \mathbf{u} \quad \text { in } \mathcal{V}_{2}^{1,0}\left(\mathcal{Q}_{T}\right)
$$

\section{The free boundary problem}

The goal of this section is to prove the local existence of solutions for problem (1.1a)-(1.4). In this section, let

$$
\begin{aligned}
& T_{0}:=\min \left\{\left(\frac{\delta_{0}}{4}\right)^{2 / 3},\left[C_{4}\left(\frac{2}{\delta_{0}},\left(\frac{\delta_{0}}{4}\right)^{1 / 9}\right) \sum_{i=1}^{n} \frac{\hat{a}^{i}}{m^{i}}\right]^{-12}\right\}, \quad 0<T \leq T_{0}, \\
& \mathbb{B}:=\left\{\mathbf{r}(t)=\left(r^{1}(t), \ldots, r^{n}(t)\right):\|\mathbf{r}(t)\|_{\mathbf{L}^{3}(0, T)} \leq 1\right\} .
\end{aligned}
$$

Then, for $\mathbf{r}(t) \in \mathbb{B}$, we have

$$
\begin{aligned}
& \int_{0}^{T}\left|r^{i}(t)\right| \mathrm{d} t \leq T^{2 / 3}\left\|r^{i}(t)\right\|_{L^{3}(0, T)} \leq T^{2 / 3} \leq \delta_{0} / 4, \\
& \int_{0}^{T}\left|r^{i}(t)-r^{i-1}(t)\right| \mathrm{d} t \leq 2 T^{2 / 3} \leq \delta_{0} / 2, \quad i=1, \ldots, n .
\end{aligned}
$$

Thus $\varphi^{i}(t)=d^{i}+\int_{0}^{t} r^{i}(t) \mathrm{d} t, i=1, \ldots, n$, satisfy

$$
\varphi^{1}(t) \geq d^{1}-\int_{0}^{T}\left|r^{1}(t)\right| \mathrm{d} t \geq \frac{3}{4} \delta_{0}, \quad d-\varphi^{n}(t) \geq d-d^{n}-\int_{0}^{T}\left|r^{n}(t)\right| \mathrm{d} t \geq \frac{3}{4} \delta_{0},
$$

and

$$
\varphi^{i}(t)-\varphi^{i-1}(t) \geq d^{i}-d^{i-1}-\int_{0}^{T}\left|r^{i}(t)-r^{i-1}(t)\right| \mathrm{d} t \geq \frac{\delta_{0}}{2}, \quad i=2, \ldots, n .
$$


For $\mathbf{r}(t) \in \mathbb{B}$, define

$$
\mathcal{G}(\mathbf{r})(t)=\left(-\frac{\hat{a}^{1} u_{x}^{1-}\left(\varphi^{1}(t), t\right)}{u^{1}\left(\varphi^{1}(t), t\right)}, \ldots,-\frac{\hat{a}^{n} u_{x}^{n-}\left(\varphi^{n}(t), t\right)}{u^{n}\left(\varphi^{n}(t), t\right)}\right) \quad \text { for a.e. } t \in[0, T]
$$

where $\varphi^{i}(t)=d^{i}+\int_{0}^{t} r^{i}(\tau) \mathrm{d} \tau(i=1, \ldots, n)$ and $\mathbf{u}$ is the solution in $\mathscr{S}$ of problem (1.1a)-(1.3) associated with $\varphi(t)=\left(\varphi^{1}(t), \ldots, \varphi^{n}(t)\right)$.

Proof of Theorem 2.2 By a slight modification, we use the methods and the framework of $[3,5]$ to prove Theorem 2.2 . We only sketch the main steps and omit most of the detailed proofs.

Step 1. We will show that $\mathcal{G}(\mathbb{B}) \subseteq \mathbb{B}$.

Employing (4.1), (4.2), (2.9) and Hölder?s inequality, we get

$$
\left\|\varphi_{t}\right\|_{\mathbf{L}^{2}(0, T)} \leq\left\|\boldsymbol{\varphi}_{t}\right\|_{\mathbf{L}^{3}(0, T)} T_{0}^{1 / 6} \leq\left(\delta_{0} / 4\right)^{1 / 9}
$$

and

$$
\begin{aligned}
\|\mathcal{G}(\mathbf{r})(t)\|_{\mathbf{L}^{3}(0, T)} & =\sum_{i=1}^{n}\left\|\frac{\hat{a}^{i} u_{x}^{i-}\left(\varphi^{i}(t), t\right)}{u^{i}\left(\varphi^{i}(t), t\right)}\right\|_{L^{3}(0, T)} \\
& \leq \sum_{i=1}^{n} \frac{\hat{a}^{i}}{m^{i}}\left(\int_{0}^{T}\left\|u_{x}^{i}\right\|_{L^{\infty}\left(0, \varphi^{i}(t)\right)}^{3} \mathrm{~d} t\right)^{1 / 3} \\
& \leq \sum_{i=1}^{n} \frac{\hat{a}^{i}}{m^{i}}\left\|u_{x}^{i}\right\|_{L^{\infty, 4}\left(Q_{T}\right)} T^{1 / 12} \\
& \leq T_{0}^{1 / 12} C_{4}\left(\frac{2}{\delta_{0}},\left(\frac{\delta_{0}}{4}\right)^{1 / 9}\right) \sum_{i=1}^{n} \frac{\hat{a}^{i}}{m^{i}} \\
& \leq 1 .
\end{aligned}
$$

Consequently, $\mathcal{G}(\mathbb{B}) \subseteq \mathbb{B}$.

Step 2. By (2.11) and a slight modification of the proof of [3, Proposition 3.4], we can prove that $\mathcal{G}: \mathbb{B} \rightarrow \mathbb{B}$ is continuous and $\mathcal{G}(\mathbb{B}) \subset \mathbb{B}$ is precompact in $\mathbf{L}^{3}(0, T)$.

Step 3. It follows from Schauder?s fixed point theorem that $\mathcal{G}: \mathbb{B} \rightarrow \mathbb{B}$ has at least one fixed point $\mathbf{r}(t)$. Then the curves $\varphi^{i}(t)=d^{i}+\int_{0}^{t} r^{i}(\tau) \mathrm{d} \tau, i=1, \ldots, n$, and the vector function $\mathbf{u}(x, t)$ associated with $\varphi(t)$ by Theorem 2.1 satisfy (1.4) for almost all $t \in[0, T]$. Moreover, (1.4), (2.6) and (2.9) imply that $\varphi(t)$ is in $\mathbf{W}_{4}^{1}(0, T)$.

Step 4. Using the regularity result of Theorem 2.1(iii) and the approximation result of Corollary 3.3, and calculating $\int_{0}^{T} \frac{\mathrm{d}}{\mathrm{d} t} \int_{0}^{d}\left(A^{l}(x, t)\right)^{3}\left(u_{x}^{l}\right)^{4} \mathrm{~d} x \mathrm{~d} t$, we can conclude from the proof similar to that of $[5$, Lemma 2.5$]$ that

$$
\sup _{[0, T]} \int_{0}^{d}\left(u_{x}^{l}\right)^{4} \mathrm{~d} x \leq C\left(1 / \delta_{0}, T_{0}\right), \quad l=1, \ldots, N
$$

In addition, for each $l=n+1, \ldots, N, u^{l}$ satisfies the linear equation

$$
u_{t}^{l}-a^{l} u_{x x}^{l}=\underline{f}^{l}(x, t):=b^{l}\left(u^{l}\right) u_{x}^{l}+G^{l}(x, t, \mathbf{u}) \quad\left((x, t) \in Q_{T}\right)
$$


Estimate (4.3) yields $f^{l}(x, t) \in L^{4}\left(Q_{T}\right)$. Thus, according to the local parabolic $L^{p}$ estimates, we get $u^{l} \in W_{4}^{2,1}\left(Q^{\prime}\right)$ for any interior domain $Q^{\prime}$ of $Q_{T}$. This implies that

$$
u_{x}^{l} \in C^{\alpha, \alpha / 2}\left(\bar{Q}^{\prime}\right)
$$

for some $\alpha \in(0,1)$.

Step 5. By (4.3), (4.4) and hypothesis (H)(iii), a similar argument as that for [5, Theorem 2.6] shows that for each $l=1, \ldots, n, \varphi^{l}(t) \in C^{2+\alpha}((0, T])$. Then (1.4) holds for $t \in(0, T]$ and $\{\mathbf{u}(x, t), \varphi(t)\}$ is a local solution of problem (1.1a)-(1.4).

Remark 4.1 Theorem 2.2 concerns the local existence of solutions for (1.1a)-(1.4). For the case $n=1$, the global existence of solutions of (1.1a)-(1.4) can be proved by the same argument as that in [4]. For the case $n>1$, the difficulty to prove the global existence of solutions may be that the free boundaries can intersect with each other.

\section{An example}

In this section, we give an example satisfying the conditions in hypothesis $(\mathrm{H})$.

Consider the Lotka-Volterra reaction-diffusion model with two predators $u^{1}, u^{2}$, and one prey $u^{3}$, where each of predators $u^{1}, u^{2}$ has two groups and the prey $u^{3}$ is native and has only one group. Then the reaction-diffusion functions are defined by

$$
\left\{\begin{array}{l}
g_{k}^{1}(\mathbf{u})=\gamma_{k}^{1} u^{1}\left(1-\beta_{k}^{1} u^{1}-\varsigma_{k}^{1} u^{2}+\sigma_{k}^{1} u^{3}\right), \\
g_{k}^{2}(\mathbf{u})=\gamma_{k}^{2} u^{2}\left(1-\beta_{k}^{2} u^{2}-\varsigma_{k}^{2} u^{1}+\sigma_{k}^{2} u^{3}\right), \\
g_{k}^{3}(\mathbf{u})=\gamma^{3} u^{3}\left(1-\beta^{3} u^{3}-\varsigma_{k}^{3} u^{1}-\sigma_{k}^{3} u^{2}\right), \quad k=1,2,3,
\end{array}\right.
$$

where $\gamma_{k}^{l}, \beta_{k}^{l}, \varsigma_{k}^{l}, \sigma_{k}^{l}(l, k=1,2), \gamma^{3}, \beta^{3}, \varsigma_{3}^{3}$ and $\sigma_{3}^{3}$ are all positive constants. For the mankind?s influence, we assume that

$$
\left\{\begin{array}{l}
\hat{b}\left(u^{l}\right)=\hat{\chi}^{l} u^{l}, \quad \tilde{b}\left(u^{l}\right)=-\tilde{\chi}^{l} u^{l}, \quad l=1,2, \\
b^{3}\left(u^{3}\right)=\chi^{3} u^{3},
\end{array}\right.
$$

where $\hat{\chi}^{l}, \tilde{\chi}^{l}(l=1,2)$ and $\chi^{3}$ are constants. Thus $\mathbf{u}=\left(u^{1}, u^{2}, u^{3}\right)$ and $\varphi=\left(\varphi^{1}, \varphi^{2}\right)$ are governed by system (1.1a)-(1.4), where $n=2, N=3$ and the coefficients are replaced by (5.1), (5.2).

Assume that

$$
\begin{aligned}
& \max _{k=1,2,3}\left\{\frac{1+\sigma_{k}^{1}\left(\beta^{3}\right)^{-1}}{\beta_{k}^{1}}, \frac{1+\sigma_{k}^{2}\left(\beta^{3}\right)^{-1}}{\beta_{k}^{2}}\right\}<\min _{k=1,2,3}\left\{\frac{1}{\varsigma_{k}^{1}}, \frac{1}{\varsigma_{k}^{2}}, \frac{1}{\varsigma_{k}^{3}+\sigma_{k}^{3}}\right\}, \\
& 0<\psi^{l}(x, t)<\min _{k=1,2,3}\left\{\frac{1}{\varsigma_{k}^{1}}, \frac{1}{\zeta_{k}^{2}}, \frac{1}{\zeta_{k}^{3}+\sigma_{k}^{3}}\right\} \quad\left((x, t) \in S_{T} \cup([0, d] \times\{0\})\right), l=1,2,
\end{aligned}
$$

and

$$
0<\psi^{3}(x, t)<\left(\beta^{3}\right)^{-1}
$$


We will look for $\mathbf{M}=\left(M^{1}, M^{2}, M^{3}\right)$ and $\mathbf{m}=\left(m^{1}, m^{2}, m^{3}\right)$ satisfying (2.1). This requires that

$$
\begin{aligned}
& \left\{\begin{array}{l}
1-\beta_{k}^{1} M^{1}-\varsigma_{k}^{1} m^{2}+\sigma_{k}^{1} M^{3} \leq 0, \\
1-\beta_{k}^{2} M^{2}-\varsigma_{k}^{2} m^{1}+\sigma_{k}^{2} M^{3} \leq 0, \\
1-\beta^{3} M^{3}-\varsigma_{k}^{3} m^{1}-\sigma_{k}^{3} m^{2} \leq 0, \quad k=1,2,3,
\end{array}\right. \\
& \left\{\begin{array}{l}
1-\beta_{k}^{1} m^{1}-\varsigma_{k}^{1} M^{2}+\sigma_{k}^{1} m^{3} \geq 0, \\
1-\beta_{k}^{2} m^{2}-\varsigma_{k}^{2} M^{1}+\sigma_{k}^{2} m^{3} \geq 0, \\
1-\beta^{3} m^{3}-\varsigma_{k}^{3} M^{1}-\sigma_{k}^{3} M^{2} \geq 0, \quad k=1,2,3,
\end{array}\right.
\end{aligned}
$$

and

$$
m^{l}<\psi^{l}(x, t)<M^{l} \quad\left((x, t) \in S_{T} \cup\{\Omega \times\{0\}\}\right), l=1,2,3 .
$$

Choose $m^{1}=m^{2}=m^{3}$ and $M^{1}=M^{2}$. It is obvious that (5.6) and (5.7) hold if

$$
\left\{\begin{array}{l}
1-\beta_{k}^{1} M^{1}+\sigma_{k}^{1} M^{3} \leq 0 \\
1-\beta_{k}^{2} M^{1}+\sigma_{k}^{2} M^{3} \leq 0 \\
1-\beta^{3} M^{3} \leq 0, \quad k=1,2,3
\end{array}\right.
$$

and

$$
\left\{\begin{array}{l}
1-\beta_{k}^{1} m^{1}-\varsigma_{k}^{1} M^{1} \geq 0 \\
1-\beta_{k}^{2} m^{1}-\varsigma_{k}^{2} M^{1} \geq 0 \\
1-\beta^{3} m^{1}-\varsigma_{k}^{3} M^{1}-\sigma_{k}^{3} M^{1} \geq 0, \quad k=1,2,3 .
\end{array}\right.
$$

By (5.3)-(5.5), we can first choose $m^{1}=m^{2}=m^{3}$ small enough such that

$$
\begin{gathered}
0<m^{l}<\psi^{l}(x, t)<\min _{k=1,2,3}\left\{\frac{1-\beta_{k}^{1} m^{1}}{\varsigma_{k}^{1}}, \frac{1-\beta_{k}^{2} m^{1}}{\varsigma_{k}^{2}}, \frac{1-\beta_{k}^{3} m^{1}}{\varsigma_{k}^{3}+\sigma_{k}^{3}}\right\} \\
\left((x, t) \in S_{T} \cup([0, d] \times\{0\})\right), l=1,2,
\end{gathered}
$$

and

$$
\max _{k=1,2,3}\left\{\frac{1+\sigma_{k}^{1}\left(\beta^{3}\right)^{-1}}{\beta_{k}^{1}}, \frac{1+\sigma_{k}^{2}\left(\beta^{3}\right)^{-1}}{\beta_{k}^{2}}\right\}<\min _{k=1,2,3}\left\{\frac{1-\beta_{k}^{1} m^{1}}{\varsigma_{k}^{1}}, \frac{1-\beta_{k}^{2} m^{1}}{\varsigma_{k}^{2}}, \frac{1-\beta_{k}^{3} m^{1}}{\varsigma_{k}^{3}+\sigma_{k}^{2}}\right\} .
$$

Furthermore, choose $M^{1}, M^{2}$ and $M^{3}$ such that

$$
\begin{aligned}
& M^{3}=\left(\beta^{3}\right)^{-1}, \\
& M^{1}=M^{2} \geq \max _{k=1,2,3}\left\{\frac{1+\sigma_{k}^{1}\left(\beta^{3}\right)^{-1}}{\beta_{k}^{1}}, \frac{1+\sigma_{k}^{2}\left(\beta^{3}\right)^{-1}}{\beta_{k}^{2}}\right\}, \\
& M^{1}=M^{2}>\psi^{l}(x, t) \quad\left((x, t) \in S_{T} \cup([0, d] \times\{0\})\right), \quad l=1,2,
\end{aligned}
$$

and

$$
M^{1}=M^{2} \leq \min _{k=1,2,3}\left\{\frac{1-\beta_{k}^{1} m^{1}}{\varsigma_{k}^{1}}, \frac{1-\beta_{k}^{2} m^{1}}{\varsigma_{k}^{2}}, \frac{1-\beta_{k}^{3} m^{1}}{\varsigma_{k}^{3}+\sigma_{k}^{2}}\right\} .
$$


The relations (5.8)-(5.10) are guaranteed by the above $\mathbf{M}$ and $\mathbf{m}$. Hence $\mathbf{M}$ and $\mathbf{m}$ satisfy (5.6)-(5.8); and consequently, $\mathbf{M}$ and $\mathbf{m}$ satisfy (2.1). Moreover, $\mathbf{g}_{k}(\mathbf{u})=\left(g_{k}^{1}(\mathbf{u}), g_{k}^{2}(\mathbf{u}), g_{k}^{3}(\mathbf{u})\right)$, $k=1,2,3$, are mixed quasimonotone in $\mathscr{S}$ with the same index vector $\left(\left(u^{3}\right) ;\left(u^{3}\right) ;[\mathbf{u}]_{0}\right)$. The above argument shows that the conditions in hypothesis $(\mathrm{H})$ can be satisfied. Then we get the following corollary from Theorem 2.2 .

Corollary 5.1 In problem (1.1a)-(1.4), let $n=2, N=3$, and let the coefficients be given by (5.1), (5.2). Assume that the conditions in (5.3)-(5.5) and hypothesis $(\mathrm{H})(\mathrm{i})$ are satisfied. Then there exist $T>0$ and a pair of functions $\mathbf{u}(x, t), \varphi(t)$ such that $\{\mathbf{u}(x, t), \varphi(t)\}$ is a solution of (1.1a)-(1.4).

\section{Competing interests}

The author declares that they have no competing interests.

\section{Acknowledgements}

The author would like to thank the reviewers and the editors for their valuable suggestions and comments. The work was supported by the research fund of Department of Education of Sichuan Province and the research fund of Chengdu Normal University (CS14ZD01).

Received: 11 June 2014 Accepted: 8 December 2014 Published online: 10 January 2015

\section{References}

1. Kim, K, Lin, Z: A free boundary problem for a parabolic system describing an ecological model. Nonlinear Anal., Real World Appl. 10, 428-436 (2009)

2. Kamynin, LI: On the linear Verigin problem. Dokl. Akad. Nauk SSSR 150, 1210-1213 (1963) (in Russian)

3. Evans, LC: A free boundary problem: the flow of two immiscible fluids in a one-dimensional porous medium, I. Indiana Univ. Math. J. 26, 914-932 (1977)

4. Evans, LC: A free boundary problem: the flow of two immiscible fluids in a one-dimensional porous medium, II. Indiana Univ. Math. J. 27, 93-111 (1978)

5. Evans, LC, Friedman, A: Regularity and asymptotic behavior of two immiscible fluids in a one-dimensional porous medium. J. Differ. Equ. 31, 366-391 (1979)

6. Liang, J: The one-dimensional quasilinear Verigin problem. J. Partial Differ. Equ. 4, 74-96 (1991)

7. Mimura, M, Yamada, Y, Yotsutani, S: A free boundary problem in ecology. Jpn. J. Appl. Math. 2, 151-186 (1985)

8. Ling, Z, Tang, Q, Lin, Z: A free boundary problem for two-species competitive model in ecology. Nonlinear Anal., Real World Appl. 11, 1775-1781 (2010)

9. Lin, Z: A free boundary problem for a predator prey model. Nonlinearity 20, 1883-1892 (2007)

10. Guan, Z: A class of free boundary problems to quasi-linear parabolic equations. Acta Math. Appl. Sin. 15, 197-205 (1999)

11. Yi, FH: Global classical solution of Muskat free boundary problem. J. Math. Anal. Appl. 288, 442-461 (2003)

12. Merz, W, Rybka, P: A free boundary problem describing reaction-diffusion problems in chemical vapor infiltration of pyrolytic carbon. J. Math. Anal. Appl. 292, 571-588 (2004)

13. Yi, F: One dimensional combustion free boundary problem. Glasg. Math. J. 46, 63-75 (2004)

14. Boyadjiev, G, Kutev, N: Diffraction problems for quasilinear reaction-diffusion systems. Nonlinear Anal. 55, 905-926 (2003)

15. Tan, QJ: Systems of quasilinear parabolic equations with discontinuous coefficients and continuous delays. Adv. Differ. Equ. 2011, Article ID 925173 (2011). doi:10.1155/2011/925173

16. Tan, QJ, Pan, CY: Diffraction problems for quasilinear parabolic systems with boundary intersecting interfaces. Bound. Value Probl. 2013, Article ID 99 (2013)

17. Ladyzenskaya, OA, Solonnikov, VA, Ural?ceva, NN: Linear and Quasilinear Equations of Parabolic Type. Am. Math. Soc., Providence (1968)

18. Tan, QJ, Leng, ZJ: The regularity of generalized solutions for the $n$-dimensional quasi-linear parabolic diffraction problems. Nonlinear Anal. 69, 4624-4642 (2008) 